\title{
OPTIMAL STABILIZATION POLICY WITH SEARCH EXTERNALITIES
}

\author{
Aleksander Berentsen \\ University of Basel \\ and \\ Federal Reserve Bank of St. Louis
}

Christopher Waller

Federal Reserve Bank of St. Louis

and

University of Notre Dame

\begin{abstract}
We study optimal monetary stabilization policy in a DSGE model with microfounded money demand. A search externality creates "congestion," which causes aggregate output to be inefficient. Because of the informational frictions that give rise to money, households are unable to insure themselves perfectly against aggregate shocks. This gives rise to a welfare-improving role for monetary policy that works by adjusting the nominal interest rate in response to these shocks. Optimal policy is determined by choosing a set of state-contingent nominal interest rates to maximize the expected lifetime utility of the agents subject to the constraints of being an equilibrium.
\end{abstract}

Keywords: Monetary Policy, Optimal Stabilization Policy, Search Equilibrium, Microfoundation of Money

\section{INTRODUCTION}

Since Keynes, monetary economists have been interested in studying stabilization policy in the presence of market frictions. The typical friction imposed is nominal price and/or wage rigidity. This assumption means that changes in the money supply are not neutral; consequently monetary policy can affect real allocations. In this class of models, the standard policy implication is to conduct countercyclical monetary policy. The intuition for this is straightforward-if demand for goods increases, then it is efficient to let prices rise to some extent. But with sticky prices, households consume more than the efficient amount. Thus, the monetary authority

\footnotetext{
The views expressed are those of the individual authors and do not necessarily reflect official positions of the Federal Reserve Bank of St. Louis, the Federal Reserve System, or the Board of Governors. The paper has benefitted from comments by participants at several seminar and conference presentations-in particular Sanjay Chugh, Allen Head, and Huberto Ennis. We thank the Federal Reserve Banks of Cleveland, Minneapolis, and St. Louis, CES in Munich, and the Kellogg Institute at the University of Notre Dame for research support. Berentsen acknowledges support by the Bank for International Settlements (the usual disclaimer applies). Address correspondence to: Aleksander Berentsen, Economics Department, University of Basel, Peter-Merian-Weg 6, Postfach, CH-4002 Basel, Switzerland; e-mail: aleksander.berentsen@unibas.ch.
} 
should tighten policy by raising the nominal interest rate to constrain consumption from rising to inefficiently high levels.

An interesting question is whether this same policy advice holds when other frictions are the reason for the non-neutrality of money. For example, in searchbased models of money or in cash-in-advance models, households face liquidity constraints on purchases of goods. In these models, should the monetary authority pursue a countercyclical monetary policy? Ireland (1996), for example, shows that the Friedman rule is optimal and perfectly stabilizes demand shocks. But what if the Friedman rule is not optimal because of some other friction in the economy? What do optimal stabilization policies look like? These are the questions we address in this paper.

The basic framework is that of Lagos and Wright (2005), where informational frictions make money essential as a medium of exchange. ${ }^{1}$ We modify the model in several ways. First, we introduce a search externality by assuming that in every period producers make an entry decision. Second, we carefully model the existence of a credit market that allows agents to borrow and lend money. Third, we introduce a variety of well-defined aggregate shocks, such as productivity and preference shocks, that generate consumption risk for households. Finally, we consider three pricing protocols - competitive pricing, monopoly pricing, and price posting —in the market where money is essential as a medium of exchange.

Borrowing from Rocheteau and Wright (2005), we assume that upon entering the market, a producer is able to trade with some probability, which may not be one. In short, he may be shut out of the market despite having paid the entry cost. We then study optimal stabilization under two assumptions regarding this trading probability. In the first case, we assume that the trading probability is independent of the number of producers in the market. In the second case, we assume that the probability of trading is decreasing in the number of entering producers. This is intended to capture the idea that as more producers enter, congestion occurs, making it harder to trade and earn profits.

Our basic results concerning the optimal stabilization policy are as follows. With a fixed probability of trading, the optimal monetary policy is to run the Friedman rule and set the nominal interest rate to zero in all states. This is true for all three pricing protocols. When the trading probability depends on aggregate entry, a congestion externality arises that makes entry inefficiently high. Thus, the central bank finds it optimal to raise interest rates above zero in all states in order to reduce profits and deter entry, even though it lowers average consumption. Once again, this is true for all pricing protocols. In short, the zero lower bound is never a binding constraint in our model. The key to implementing the desired allocation is to manipulate the relative prices of goods across markets by choosing state-dependent nominal interest rates.

Our framework for studying optimal stabilization policy builds on Berentsen and Waller (2011). In this earlier paper, we have shown that even when all prices in the economy are fully flexible, monetary injections are non-neutral if the central bank has a price-level target. Furthermore, we have demonstrated that away from the 
Friedman rule there is a welfare-improving role for stabilization policy. However, we have also found that the optimal policy is the Friedman rule, in which case the optimal stabilization policy requires the central bank to set a nominal interest rate of zero in all states. The key innovation of our current paper is that we introduce the previously mentioned congestion effect. With this externality, the optimal policy deviates from the Friedman rule and allows for a nontrivial optimal stabilization policy. Furthermore, we study how different pricing protocols affect the optimal policy, whereas in our earlier paper we only consider competitive pricing.

There are precedents for such macro externalities in the literature. For example, in endogenous entry/search models where the terms of trade are determined by bargaining, there may be too many buyers or sellers relative to the social optimum. In these models, deviating from the Friedman rule may be optimal to improve the extensive margin. Such externalities are studied, for example, in Shi (1997), Lagos and Rocheteau (2005), Rocheteau and Wright (2005), Aruoba et al. (2007), Berentsen et al. (2007), Aruoba and Chugh (2010), and Liu et al. (2011). Although these papers investigate the implications of these externalities for the optimal steady-state inflation rate, we investigate the implications for the optimal stabilization policy. $^{2}$

Our framework for studying optimal stabilization policy is also related to but substantially different from the literature on endogenous entry. Jaimovich and Floetotto (2008) use a prototypical real business cycle model; hence there is no role for monetary policy. The other papers in this area are based on New Keynesian sticky price models. Furthermore, many of them look at the effects of monetary shocks - they do not study optimal monetary policy. Of those that study optimal policy, Bilbiie et al. (2007) and Bergin and Corsetti (2008) do so for a simple class of interest rate rules with a single productivity shock. They consider a model where entry enlarges the set of goods available to households. The households have a love for variety, so that enlarging the set of goods can cause a positive externality on household utility. In contrast, in our model firm entry imposes a negative congestion externality. ${ }^{3}$ As such, our model captures episodes where there was "too much entry from a social point of view," as suggested by many observers of the recent events in the housing and commercial property markets. Finally, Lewis (2009) is also related to our work in that she derives the optimal monetary policy using a Ramsey primal approach in a cash-in-advance model. She finds that the Friedman rule is optimal and that under nominal rigidities monetary policy has a stabilization role through its control over the money stock. Finally, we address other issues, such as the zero lower nominal bound on interest rates, that these papers do not.

Finally, we want to address one caveat. Our results rely on the assumption that fiscal policy is absent or impotent as an instrument to control entry. Correia et al. (2008) show in monetary economies with sticky prices that the efficient allocation can be implemented if fiscal and monetary policies are chosen optimally. In our environment, if the government were to design the tax system so that all entry externalities were internalized, the Friedman rule would implement the first-best 
allocation. However, this would require that taxes be state-contingent, which might be difficult to implement in practice. Furthermore, in any of the papers mentioned in the preceding, if the government has a sufficient set of tax instruments, the efficient allocation can be restored.

The paper proceeds as follows. In Section 2, we describe the environment and derive the first-best allocation. In Section 3, we present the agents' decision problems. Section 4 contains the central bank's maximization problem and the optimal monetary policy for each pricing protocol. Section 5 concludes.

\section{THE ENVIRONMENT}

Time is discrete and continues forever. ${ }^{4}$ In each period three perfectly competitive markets open sequentially. The first market is a competitive credit market and the third market is a competitive goods market. The second market is also a goods market, for which we study various market structures. There is a continuum of two types of agents, called households and sellers. They differ in terms of when they produce and consume, as follows. All agents can produce and consume a perishable good in the last market. In the second market, households can consume but cannot produce and sellers can produce but cannot consume. We assume that all trades in the second market are anonymous, which rules out trade credit. Because all agents are anonymous and there is a double coincidence problem, sellers require immediate compensation. So households must pay with money in market 2 , generating an essential role for money. ${ }^{5}$

The instantaneous utility of a household at date $t$ is

$$
U_{t}^{b}=v\left(x_{t}\right)-y_{t}+\psi_{t} u\left(q_{t}^{b}\right)
$$

where $x_{t}$ is consumption and $y_{t}$ production in the last market. ${ }^{6}$ The quantity $q_{t}^{b}$ is a household's consumption in the second market and $\psi \geq 0$ is a preference parameter. We assume $u^{\prime}>0, u^{\prime \prime}<0, u^{\prime}(0)=+\infty$, and $u^{\prime}(\infty)=0$. Furthermore, we assume the coefficient of relative risk aversion, $R^{u} \equiv-q^{b} u^{\prime \prime} / u^{\prime}$, is constant and less than one. ${ }^{7}$ In the last market, the utility function satisfies $v^{\prime}>0, v^{\prime \prime}<0$, $v^{\prime}(0)=\infty$, and there is an $x^{*}$ such that $v^{\prime}\left(x^{*}\right)=1$.

The instantaneous utility of a seller at date $t$ is

$$
U_{t}^{s}=v\left(x_{t}\right)-y_{t}-\left(1 / \alpha_{t}\right) c\left(q_{t}\right)
$$

where $q_{t}$ denotes the seller's production in the second market. Production disutility satisfies $c^{\prime}, c^{\prime \prime}, c^{\prime \prime \prime} \geq 0$, and $c(0)=c^{\prime}(0)=0$. Denote the elasticity of marginal cost as $R^{c} \equiv q c^{\prime \prime} / c^{\prime}$. The parameter $\alpha$ is a productivity parameter measured in utility terms, where higher values of $\alpha$ are associated with higher productivity and thus lower marginal utility costs of production. The discount factor across dates is $\beta=1 /(1+r) \in(0,1)$, where $r$ is the time rate of discount. 


\subsection{Credit Market}

At the beginning of a period, each household receives an idiosyncratic preference shock $\psi_{t} \in\{0, \varepsilon\}$ with $\varepsilon>0$. The probability that $\psi_{t}=\varepsilon$ is $1 / 2$, meaning that there is an equal probability that a household wants to consume or not in market $2 .^{8}$ We call households that consume "buyers," and those that do not "nonbuyers." These preference shocks generate an ex post inefficiency because nonbuyers are holding idle balances, whereas buyers are cash-constrained. This inefficiency generates a welfare-improving role for a credit market where households can borrow or lend money at the nominal interest rate $i$.

Although the goods trade is anonymous, we assume the existence of a recordkeeping technology for financial transactions as in Berentsen et al. (2007). In all models with credit, default is a serious issue. To focus on optimal stabilization, we simplify the analysis by assuming that some mechanism exists that ensures the repayment of loans in the third market. ${ }^{9}$ One can show that because of the quasilinearity of preferences in market 3 , there is no gain from multiperiod contracts. Furthermore, because the states are revealed prior to contracting, the one-period nominal debt contracts that we consider are optimal.

\subsection{Shocks}

To study the optimal response to shocks, we assume that $\alpha_{t}$ and $\varepsilon_{t}$ are stochastic. The random variable $\alpha_{t}$ has support $[\underline{\alpha}, \bar{\alpha}], 0<\underline{\alpha}<\bar{\alpha}<\infty$, and $\varepsilon_{t}$ has the support $[\underline{\varepsilon}, \bar{\varepsilon}], 0<\underline{\varepsilon}<\bar{\varepsilon}<\infty$. Let $\omega_{t}=\left(\alpha_{t}, \varepsilon_{t}\right) \in \Phi$ be the state in market 1 , where $\Phi=[\underline{\alpha}, \bar{\alpha}] \times[\underline{\varepsilon}, \bar{\varepsilon}]$ is a closed and compact subset on $\boldsymbol{R}_{+}^{2}$. We allow the shocks to be serially correlated. Let $\Omega_{t}=\left\{\omega_{t}, \omega_{t-1}, \ldots\right\}$ denote the history of the aggregate state up to period $t$. For notational simplicity let $d F\left(\omega_{t} \mid \Omega_{t-1}\right) \equiv d F\left(\alpha_{t} \mid \Omega_{t-1}\right) d F\left(\varepsilon_{t} \mid \Omega_{t-1}\right)$ denote the conditional density function of $\omega_{t}$, where $d F\left(\omega_{t} \mid \Omega_{t-1}\right)=f\left(\omega_{t} \mid \Omega_{t-1}\right) d \omega_{t}$. For discussion purposes, we label $\varepsilon_{t}$ as a "demand" shock, whereas a shock to $\alpha_{t}$ is referred to as a "supply" shock.

\subsection{Free Entry and Search Frictions}

Entry is costly for sellers. At the beginning of every period after they observe the shock, sellers have to pay the cost $\kappa>0$ in terms of disutility to enter the second market. ${ }^{10}$ The set of potential sellers is denoted $\mathcal{F}$. Let $\mathcal{S} \subseteq \mathcal{F}$ denote the set of sellers that pay the utility cost $\kappa$ to enter the second market. We assume that the set of potential sellers $\mathcal{F}$ is so large that $\mathcal{S} \subset \mathcal{F}$. Let $s$ denote the measure of $\mathcal{S}$. The set of households is denoted by $\mathcal{H}$, whose size is normalized to 2 . Let $\mathcal{B} \subset \mathcal{H}$ denote the set of households with $\psi=\varepsilon$ (the buyers), where $b=1$ is the measure of $\mathcal{B}$.

We introduce search frictions along the lines of Rocheteau and Wright (2005), who assume that not all sellers that pay the fixed utility cost can trade in market 2. 
That is, paying $\kappa$ means entry into the group $\mathcal{S}$ of sellers that try to enter market 2. Only $\tilde{\mathcal{S}} \subseteq S$ succeed. Denote as $\sigma(s)$ the probability of trading in market 2 for a seller that has paid the utility cost. Then $\sigma(s) s$ is the measure of $\tilde{\mathcal{S}}$. We impose the usual assumptions on $\sigma(s)$, namely $\sigma^{\prime}(s) \leq 0, \sigma^{\prime \prime}(s) \geq 0, \sigma(s) \leq 1, \sigma(0)=1$, and $\sigma(\infty)=0$. Finally, denote as $\Sigma^{\sigma} \equiv s \sigma^{\prime}(s) / \sigma(s)<0$ the elasticity of $\sigma(s)$. As is standard in the search literature, we assume this elasticity is constant with $-\Sigma^{\sigma}<1$.

The probability of trading, $\sigma(s)$, has a natural meaning in matching models with bilateral meetings: It is the probability of having a match. In competitive environments it still captures search frictions by assuming that, although there is a competitive market, not all firms get the chance to trade in this market [see Rocheteau and Wright (2005)]. With $\sigma^{\prime}(s)<0$, a seller entering the set $\mathcal{S}$ generates a negative trading externality that the optimal policy must take into account. There are precedents for such macro externalities in the literature. For example, in endogenous entry/search models where the terms of trade are determined by bargaining, there may be too many buyers or sellers relative to the social optimum, depending on the bargaining weight. In these models, deviating from the Friedman rule may be optimal to improve the extensive margin. ${ }^{11}$ The restriction that $-\Sigma^{\sigma}<$ 1 ensures that this congestion externality is not too large.

\subsection{Monetary Policy}

We assume that a central bank exists that controls the supply of fiat money. We denote the gross growth rate of the money supply as $\gamma\left(\Omega_{t}\right)$, implying that $M_{t}\left(\Omega_{t}\right)=\gamma\left(\Omega_{t}\right) M_{t-1}$, where $M_{t}\left(\Omega_{t}\right)$ denotes the quantity of money per household in market 3 in period $t$. We allow the gross growth rate, and thus $M_{t}\left(\Omega_{t}\right)$, to depend on the entire history of the economy. The central bank implements its policy by providing state-contingent lump-sum injections of money to the households. Let $\tau_{1}\left(\Omega_{t}\right) M_{t-1}$ and $\tau_{3}\left(\Omega_{t}\right) M_{t-1}$ denote the state-contingent cash injections in markets 1 and 3, where $\gamma\left(\Omega_{t}\right)=1+\tau_{1}\left(\Omega_{t}\right)+\tau_{3}\left(\Omega_{t}\right){ }^{12}$

The precise sequence of action after the shocks are observed is as follows. First, the monetary injection $\tau_{1}\left(\Omega_{t}\right) M_{-1}$ occurs. Then, households move to the credit market, where nonbuyers $(\psi=0)$ lend their idle cash and buyers $(\psi=\varepsilon)$ borrow money. Buyers and sellers then move on to market 2 and trade goods. In the third market, all financial claims are settled and the central bank injects $\tau_{3}\left(\Omega_{t}\right) M_{t-1}$ units of money per household.

\subsection{First-Best Allocation}

Our welfare criterion is given by

$$
\mathcal{V}=\sum_{t=0}^{\infty} \beta^{t} \mathcal{W}_{t}\left(\Omega_{t-1}\right)
$$


where

$$
\begin{aligned}
& \mathcal{W}_{t}\left(\Omega_{t-1}\right)=\int_{\Phi} \int_{\mathcal{H} \cup \mathcal{F}}\left\{v\left[x_{t}(j)\right]-y(j)\right\} d j d F\left(\omega_{t} \mid \Omega_{t-1}\right) \\
& -\int_{\Phi} \int_{\mathcal{S}} \kappa d j d F\left(\omega_{t} \mid \Omega_{t-1}\right) \\
& +\int_{\Phi}\left\{\int_{\mathcal{B}} \varepsilon_{t} u\left[q_{t}^{b}(j)\right] d j-\int_{\tilde{\mathcal{S}}}\left(1 / \alpha_{t}\right) c\left[q_{t}(j)\right] d j\right\} d F\left(\omega_{t} \mid \Omega_{t-1}\right) .
\end{aligned}
$$

For each $t$, the quantities $q_{t}^{b}(j)_{j \in \mathcal{B}}$ are the consumption quantities of all households with $\psi=\varepsilon$ (the buyers) and $q_{t}(j)_{j \in \tilde{\mathcal{S}}}$ are the production quantities of all sellers that pay the entry cost and are able to enter market 2 . The quantities $x_{t}(j)_{j \in \mathcal{H} \cup \mathcal{F}}$ denote the consumption quantities of all households and all sellers in market 3 , and $y_{t}(j)_{j \in \mathcal{H} \cup \mathcal{F}}$ denote the production quantities of all households and all sellers in market 3 .

An efficient allocation is defined as paths for $q_{t}^{b}(j)_{j \in \mathcal{B}}, q_{t}(j)_{j \in \tilde{\mathcal{S}}}, x_{t}(j)_{j \in \mathcal{H} \cup \mathcal{F}}$,

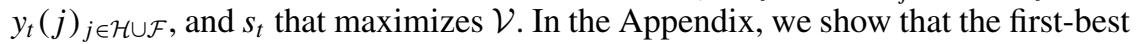
allocation is a symmetric, stationary list,

$$
\left\{x^{*}(\omega), q^{b *}(\omega), q^{*}(\omega), s^{*}(\omega)\right\}_{\omega \in \Phi},
$$

that satisfies $v^{\prime}\left[x^{*}(\omega)\right]=1, q^{b *}(\omega)=\sigma\left[s^{*}(\omega)\right] s^{*}(\omega) q^{*}(\omega)$, and $q^{*}(\omega)$ and $s^{*}(\omega)$ solve

$$
\begin{aligned}
\varepsilon u^{\prime}\left[q^{b}(\omega)\right] & =(1 / \alpha) c^{\prime}[q(\omega)], \\
\kappa & =\sigma[s(\omega)]\left(1+\Sigma^{\sigma}\right)(1 / \alpha)\left\{c^{\prime}[q(\omega)] q(\omega)-c[q(\omega)]\right\}
\end{aligned}
$$

The quantity $q(\omega)$ is a seller's production and $q^{b}(\omega)$ a buyer's consumption in market 2, and $x(\omega)$ is consumption in market 3. Equation (4) is a production efficiency condition for market 2 . It requires that the marginal consumption utility equal the marginal production disutility for each aggregate state. Equation (5) is a zero-profit condition that determines entry. It says that the entry cost must equal the expected profit in utility terms.

Note that $x^{*}(\omega)$ is not history-dependent-it depends only on the current realization of the aggregate state. The planner faces no intertemporal trade-offs and so he simply chooses the quantities that maximize welfare state by state for all $t$. This implies that the history of the shock process is irrelevant for the efficient allocation. In the Appendix, we show that the first-best allocation exists and is unique. Furthermore, comparative statics on (4)-(5) shows that $q^{*}(\omega)$ is increasing in all of the shocks, whereas $s^{*}(\omega)$ is increasing in $\varepsilon$, but is ambiguous in $\alpha .^{13}$

\section{Example 1}

To help illustrate how our model works, we use the same example throughout the paper. The functional forms are $\varepsilon u\left[q^{b}(\omega)\right]=\varepsilon q^{b}(\omega)^{1-\eta}, c[q(\omega)]=q(\omega)^{\rho} / \rho$, 
$\sigma(s)=s^{-\theta}$ with $\rho>1>\theta \geq 0$, and the entry cost is $\kappa=1$. When $\theta=0$, there is no congestion externality. With these assumptions, the planner's allocation is given by

$$
\begin{aligned}
& q^{*}(\omega)=\left(\frac{\theta \rho+\rho}{\rho-1}\right)^{\eta \delta} \alpha^{(\theta+\eta) \delta} * \varepsilon^{\theta \delta} \\
& s^{*}(\omega)=\left(\frac{\rho-1}{\theta \rho+\rho}\right)^{(\rho-1+\eta) \delta} \alpha^{(1-\eta) \delta} * \varepsilon^{\rho \delta},
\end{aligned}
$$

where $\delta \equiv[(\rho-1+\eta) \theta+\eta \rho]^{-1}$. From this example, we see that $q^{*}(\omega)$ is increasing in both shocks. We also have $s^{*}(\omega)$ increasing in $\varepsilon$ as well as $\alpha$ shocks when $\eta<1$. Thus, the planner wants entry to be procyclical. Note also that as $\rho \rightarrow 1$, costs become linear, profits go to zero, and it is optimal to have one seller producing for the entire market because entry is costly.

Why does the planner want entry to be procyclical? Consider an increase in $\varepsilon$. This implies that households want to consume more, and it is optimal to let them consume more. The planner can achieve this higher level of output by increasing the amount of goods produced by each seller, i.e., increasing $q(\omega)$, or by having more sellers enter and produce, i.e., increasing $s(\omega)$. With increasing marginal costs of production, the planner chooses to alleviate higher production costs on each individual seller by having more entry, even though it is costly. Hence, the optimal response to an $\varepsilon$ shock is to increase both the intensive and extensive margins for output. A similar argument holds for the other shock.

\section{MONETARY ALLOCATION}

Let $p_{3 t}\left(\Omega^{t}\right)$ be the time- $t$ nominal price of goods in market 3 , and thus $\phi_{t}\left(\Omega_{t}\right) \equiv$ $1 / p_{3 t}\left(\Omega_{t}\right)$ the goods price of money. We study equilibria where end-of-period real money balances are history-invariant:

$$
\phi_{t}\left(\Omega_{t}\right) M_{t}\left(\Omega_{t}\right)=\phi_{t-1}\left(\Omega_{t-1}\right) M_{t-1}\left(\Omega_{t-1}\right), \quad \forall \Omega \text {. }
$$

We refer to these as stationary equilibria. This implies that in a stationary equilibrium $\phi_{t-1}\left(\Omega_{t-1}\right) / \phi_{t}\left(\Omega_{t}\right)=\gamma\left(\Omega_{t}\right)$. In what follows, we look at a representative period $t$ and work backward from the third to the first market to examine the agents' choices. For notational ease, variables corresponding to the next period are indexed by +1 , and variables corresponding to the previous period are indexed by -1 .

\subsection{The Third Market}

In the third market, households consume $x$, produce $y$, and adjust their money balances, taking into account cash payments or receipts from the credit market. If 
a household has net borrowing of $\ell$ units of money, then it repays $(1+i) \ell$ units of money.

Consider a stationary equilibrium. Let $V_{1}(m, \Omega, t)$ denote a household's expected lifetime utility at the beginning of market 1 with $m$ money balances and history $\Omega$ in period $t$. Let $V_{3}(m, \Omega, t, \ell)$ denote a household's expected lifetime utility from entering market 3 in period $t$ with $m$ money and $\ell$ loans with history $\Omega$. For notational simplicity, in this section we suppress the dependence of the value functions on time.

Bellman's equation for a household is

$$
\begin{gathered}
V_{3}(m, \Omega, \ell)=\max _{x, y, m_{+1}}\left\{v(x)-y+\beta E\left[V_{1}\left(m_{+1}, \Omega_{+1}\right) \mid \Omega\right]\right\} \\
\text { s.t. } x+\phi m_{+1}=y+\phi\left[m+\tau_{3}(\Omega) M_{-1}\right]-\phi(1+i) \ell,
\end{gathered}
$$

where $m_{+1}$ is the money taken into period $t+1$, given the history $\Omega$. Rewriting the budget constraint in terms of $y$ and substituting into (7) yields

$$
\begin{aligned}
& V_{3}(m, \Omega, \ell)=\phi\left[m+\tau_{3}(\Omega) M_{-1}-(1+i) \ell\right] \\
& \quad+\max _{x, m_{+1}}\left\{v(x)-x-\phi m_{+1}+\beta E\left[V_{1}\left(m_{+1}, \Omega_{+1}\right) \mid \Omega\right]\right\}
\end{aligned}
$$

The first-order conditions are $v^{\prime}\left(x^{*}\right)=1$, meaning that $x^{*}$ is constant and

$$
-\phi+\beta E\left[V_{1}^{m}\left(m_{+1}, \Omega_{+1}\right) \mid \Omega\right]=0
$$

where the superscript denotes the partial derivative with respect to the argument $m$. Thus, $V_{1}^{m}$ is the marginal value of taking an additional unit of money into the first market in period $t+1$. Because the choice of $m_{+1}$ is independent of $m$, all households enter the following period with the same amount of money.

The envelope conditions are

$$
V_{3}^{m}(m, \Omega, \ell)=\phi ; \quad V_{3}^{\ell}(m, \Omega, \ell)=-\phi(1+i)
$$

As in Lagos and Wright (2005), the value function is linear in wealth.

Let $W_{1}(\epsilon, \Omega), \epsilon \in\{0,1\}$, denote a seller's expected lifetime utility at the beginning of market 1 given $\Omega$. If $\epsilon=1$, the seller has paid the entry cost $\kappa$, and if $\epsilon=0$, he has not. Note that we have also taken into account that sellers bring no money into market 1 . Because sellers do not participate in the first market, we have $W_{1}(\epsilon, \Omega)=W_{2}(\epsilon, \Omega)$. Let $W_{3}(m, \Omega)$ denote a seller's expected lifetime utility from entering market 3 with $m$ units of money given $\Omega$. Bellman's equation for a seller is

$$
\begin{aligned}
W_{3}(m, \Omega) & =\max _{x, y}\left\{v(x)-y+\beta E\left[W_{1}\left(\epsilon_{+1}, \Omega_{+1}\right) \mid \Omega\right]\right\} \\
\text { s.t. } x & =y+\phi m
\end{aligned}
$$


Rewriting the budget constraint in terms of $y$ and substituting into the objective function yields

$$
W_{3}(m, \Omega)=\phi m+\max _{x}\left\{v(x)-x+\beta E\left[W_{1}\left(\epsilon_{+1}, \Omega_{+1}\right) \mid \Omega\right]\right\} .
$$

The first-order condition is $v^{\prime}\left(x^{*}\right)=1$. The envelope condition for a seller is

$$
W_{3}^{\prime}(m, \Omega)=\phi .
$$

As was the case for households, the value function is linear in $m$.

\subsection{The Second Market}

There are three types of agents in the second market: buyers $(b)$, nonbuyers $(o)$, and sellers $(s)$. Let $V_{2}(m, \Omega, \ell, j)$ denote the value function of a household of type $j=b, o$. Let $q^{b}$ and $q$, respectively, denote the quantities consumed by a buyer and produced by a seller, and let $p$ be the nominal price of goods.

Because nonbuyers neither consume nor produce, the Bellman equation for this household is simply $V_{2}(m, \Omega, \ell, o)=V_{3}(m, \Omega, \ell)$. The one for a buyer household is

$$
\begin{gathered}
V_{2}(m, \Omega, \ell, b)=\max _{q^{b}} \varepsilon u\left(q^{b}\right)+V_{3}\left(m-p q^{b}, \Omega, \ell\right) \\
\text { s.t. } p q^{b} \leq m .
\end{gathered}
$$

Using (9), the buyer's first-order condition can be written as

$$
\lambda_{q}=\varepsilon u^{\prime}\left(q^{b}\right)-\phi p, \quad \omega \in \Phi,
$$

where $\lambda_{q}$ is the multiplier on the buyer's budget constraint. If the budget constraint is not binding, then $\varepsilon u^{\prime}\left(q^{b}\right)=\phi p$. If it is binding, then $\varepsilon u^{\prime}\left(q^{b}\right)>\phi p$ and the buyer spends all of his money; i.e., $p q^{b}=m$. In the first case, the buyer equates the marginal rate of substitution between market 2 goods and market 3 goods to the relative prices of goods in the two markets. ${ }^{14}$ In the latter case, the agent is at a "corner." In what follows, let $V_{2}^{m}(m, \Omega, \ell, j)$ denote the partial derivative of $V_{2}(m+\ell, \Omega, \ell, j)$ with respect to the first argument, $m$, and let $V_{2}^{\ell}(m, \Omega, \ell, j)$ denote the partial derivative with respect to the third argument, $\ell$.

The marginal value of a loan is the same for all households and so for $j=b, o$,

$$
V_{2}^{\ell}(m, \Omega, \ell, j)=-(1+i) \phi .
$$

Using the envelope theorem and equations (9) and (12), the marginal values of money for $j=b, o$ are

$$
\begin{aligned}
& V_{2}^{m}(m, \Omega, \ell, b)=\varepsilon u^{\prime}\left(q^{b}\right) / p, \\
& V_{2}^{m}(m, \Omega, \ell, o)=\phi .
\end{aligned}
$$


We now describe the entry behavior of the sellers in market 2. The Bellman equation for a seller who has paid the entry cost is

$W_{2}(1, \Omega)=\sigma(s) \max _{q}\left\{-(1 / \alpha) c(q)+W_{3}(p q, \Omega)\right\}+[1-\sigma(s)] W_{3}(0, \Omega)$,

subject to the pricing protocol, which we discuss in the following. The term $p q$ is the money receipts from selling output.

The Bellman equation for a seller who does not pay the entry cost satisfies $W_{2}(0, \Omega)=W_{3}(0, \Omega)$. At the beginning of the period, sellers observe the current state and the representative seller chooses to enter market 2 with probability $\pi(\Omega)$, taking the entry choices of other sellers as given. Let $\mathbf{N}$ denote the measure of potential sellers. Then, because we focus on symmetric equilibria, he expects a measure $s(\Omega)=\Pi(\Omega) \mathbf{N}$ of sellers entering, where $\Pi(\Omega)$ is the entering decision of all other sellers. Define

$$
\mathcal{D}[\Pi(\Omega) \mathbf{N}] \equiv W_{2}(1, \Omega)-W_{2}(0, \Omega)-\kappa .
$$

Equation (17) is the expected gain from entering the market. The optimal choice of $\pi$ satisfies

$$
\begin{array}{ll}
\pi(\Omega)=1 & \text { if } \mathcal{D}[\Pi(\Omega) \mathbf{N}]>0 \\
\pi(\Omega)=0 & \text { if } \mathcal{D}[\Pi(\Omega) \mathbf{N}]<0 \\
\pi(\Omega) \in[0,1] & \text { otherwise. }
\end{array}
$$

We look for symmetric Nash equilibria where all sellers choose the same entry probability $\pi(\Omega)$. Moreover, the value $(\mathrm{s})$ of $\Pi(\Omega)$ that sustain a symmetric Nash equilibrium are defined as follows:

$$
\begin{aligned}
\Pi(\Omega)=1 & \text { if } \mathcal{D}(\mathbf{N}) \geq 0, \\
\Pi(\Omega)=0 & \text { if } \mathcal{D}(0) \leq 0, \\
\mathcal{D}[\Pi(\Omega) \mathbf{N}]=0 & \text { otherwise. }
\end{aligned}
$$

Throughout the paper, we focus on equilibria where $\mathcal{D}[\Pi(\Omega) \mathbf{N}]=0$ in all states. ${ }^{15}$ Using the expressions for $W_{2}(1, \Omega)$ and $W_{2}(0, \Omega)$ and $(10)$, we then obtain the free entry condition

$$
\kappa=\sigma(s)[\phi p q-(1 / \alpha) c(q)],
$$

where the RHS is expected profits. Note that we have suppressed the dependence of $s$ and $q$ on $\omega$ for notational convenience. Because the entry cost has to be paid each period, only current profits enter into (18). Free entry requires that expected profits in market 2 equal the entry cost. Revenue after history $\Omega$, measured in utility, is given by $\phi p q$, where $p$ is the nominal price of goods in market 2 , and $\phi$ is the real 
price of money in the last market, whereas costs in utility are $-(1 / \alpha) c(q)$. Note that $\phi p=p / p_{3}$ is the relative price of goods across markets 2 and 3 .

\subsection{The Credit Market}

A household that has $m$ money at the opening of the first market has expected lifetime utility

$$
\begin{aligned}
E & {\left[V_{1}(m, \Omega)\right] } \\
& =\int_{\Phi}\left[0.5 V_{2}(m+\ell, \Omega, \ell, b)+0.5 V_{2}(m+\ell, \Omega, \ell, o)\right] d F\left(\omega \mid \Omega_{-1}\right) .
\end{aligned}
$$

Once trading types are realized, a household of type $j=b, o$ solves

$$
\max _{\ell} V_{2}(m+\ell, \Omega, \ell, j) \text { s.t. } 0 \leq m+\ell
$$

The constraint means that money holdings $m+\ell$ cannot be negative; i.e., that the household cannot lend out more money than it holds when entering the credit market. Note that there is no borrowing constraint because we assume full enforcement of repayment of loans. Thus, the first-order condition is

$$
V_{2}^{m}(m+\ell, \Omega, \ell, j)+V_{2}^{\ell}(m+\ell, \Omega, \ell, j)+\lambda(j)=0,
$$

where $\lambda(j)$ is the multiplier on the household's nonnegativity constraint. It is obvious that households with $\psi=\varepsilon$ will become borrowers, whereas those with $\psi=0$ become lenders. Consequently, we have $\lambda(b)=0$ and $\lambda(o)>0$.

Using (13)-(15), the first-order conditions can be written as

$$
\begin{aligned}
\varepsilon u^{\prime}\left(q^{b}\right) & =\phi p(1+i), \\
\lambda(o) & =i \phi
\end{aligned}
$$

To derive the expected marginal value of money at the beginning of the period, take the derivative of (19) with respect to $m$ to get

$$
E\left[V_{1}(m, \Omega)\right]=\int_{\Phi}\left\{\begin{array}{c}
0.5\left[V_{2}^{m}(m+\ell, \Omega, \ell, b)+\lambda(b)\right] \\
+0.5\left[V_{2}^{m}(m+\ell, \Omega, \ell, o)+\lambda(o)\right]
\end{array}\right\} d F\left(\omega \mid \Omega_{-1}\right) .
$$

Using (13)-(15), this expression can be written as follows:

$$
E\left[V_{1}(m, \Omega)\right]=\int_{\Phi}\left\{0.5\left[\varepsilon u^{\prime}\left(q^{b}\right) / p+\lambda(b)\right]+0.5[\phi+\lambda(o)]\right\} d F\left(\omega \mid \Omega_{-1}\right) .
$$

Finally, noting that $\lambda(b)=0$ and using equations (20) and (21), the expected marginal value of money satisfies

$$
E\left[V_{1}^{m}(m, \Omega) \mid \Omega_{-1}\right]=\int_{\Phi}\left[\varepsilon u^{\prime}\left(q^{b}\right) / p\right] d F\left(\omega \mid \Omega_{-1}\right)
$$


noting that $\Omega=\left\{\omega, \Omega_{-1}\right\}$. Differentiating (22) shows that the value function is concave in $m$. Use (8), lagged one period, to eliminate $E\left[V_{1}^{m}(m, \Omega) \mid \Omega_{-1}\right]$ from (22) Then, divide the resulting expression by $\phi_{-1}$ and rewrite to get

$$
1=\beta \int_{\Phi} \frac{\varepsilon u^{\prime}\left(q^{b}\right)}{\gamma(\Omega) p \phi} d F\left(\omega \mid \Omega_{-1}\right) .
$$

\subsection{Pricing Protocols}

We now discuss three pricing protocols: competitive pricing, state-contingent monopoly pricing, and non-state-contingent monopoly pricing. We refer to this last pricing protocol as price posting. Competitive pricing is our benchmark. We are interested in the other two protocols, because they allow us to refer our results to New Keynesian models that are characterized by monopoly pricing and sticky prices (price posting).

For each pricing protocol, we have

$$
q^{b}=\sigma(s) s q \text { for all } \omega .
$$

For competitive pricing, this is simply the market clearing condition in market 2.

For monopoly pricing, this equation also holds, because we assume a matching process that allocates $[\sigma(s) s]^{-1}$ buyers to each seller. The benefits of this matching rule are threefold. First, the first-best allocation described in Section 3 is replicated if the monopoly pricing distortion is eliminated. Second, in searchtheoretic models of money, bilateral matching creates monopoly power for both buyers and sellers in the bargaining process. This matching rule with monopoly pricing eliminates the monopsony power of the buyer and is consistent with the pricing frictions in New Keynesian models. Third, the allocation is easily compared with the flexible price allocation, because the only difference is the pricing mechanism. ${ }^{16}$

Competitive pricing. With price taking, a seller's maximization problem in market 2 is

$$
\max _{q}\left\{-(1 / \alpha) c(q)+W_{3}(p q, \Omega)\right\}
$$

Using (11), the first-order condition yields the pricing equation

$$
p=\frac{(1 / \alpha) c^{\prime}(q)}{\phi}
$$

We can then combine (20) and (25) to get an expression for the interest rate,

$$
1+i=\frac{\varepsilon u^{\prime}[\sigma(s) s q]}{(1 / \alpha) c^{\prime}(q)} .
$$


State-contingent monopoly pricing. With state-contingent monopoly pricing, because a seller faces $[\sigma(s) s]^{-1}$ buyers, the maximization problem is

$$
\begin{aligned}
& \max _{q, p}\left\{-(1 / \alpha) c(q)+W_{3}(p q, \Omega)\right\} \\
& \text { s.t. } \varepsilon u^{\prime}[\sigma(s) s q]=p \phi(1+i),
\end{aligned}
$$

where the constraint is the buyer's first-order condition for consumption. The solution yields the pricing equation ${ }^{17}$

$$
p=\frac{(1 / \alpha) c^{\prime}(q)}{\phi\left(1-R^{u}\right)} .
$$

We can then combine (20) and (27) to get an expression for the interest rate,

$$
1+i=\frac{\left(1-R^{u}\right) \varepsilon u^{\prime}[\sigma(s) s q]}{(1 / \alpha) c^{\prime}(q)} .
$$

Price posting. We now assume that sellers must set the price before the realization of the current state, $\omega$. However, they can use the information on the history of the aggregate state up to time $t-1, \Omega_{-1}$, in forming their expectations of future profits. They commit to produce whatever is demanded in state $\omega$ at the posted price, $p\left(\Omega_{-1}\right)$. However, upon seeing the shock, they can choose to enter and try to sell at the posted price. With this last assumption, no seller will experience negative expected profits at equilibrium. ${ }^{18}$ The seller's maximization problem is

$$
\begin{aligned}
& \max _{p\left(\Omega_{-1}\right)} \int_{\Phi}\left\{\begin{array}{c}
\pi(\Omega) \sigma[s(\Omega)]\left\{W_{3}\left[p\left(\Omega_{-1}\right) q(\Omega), \Omega\right]-(1 / \alpha) c[q(\Omega)]\right\} \\
+\{1-\pi(\Omega) \sigma[s(\Omega)]\} W_{3}(0, \Omega)
\end{array}\right\} \\
& \quad \times d F\left(\omega \mid \Omega_{-1}\right) \\
& \quad \text {.t. } \varepsilon u^{\prime}\{\sigma[s(\Omega)] s(\Omega) q(\Omega)\}=p\left(\Omega_{-1}\right) \phi(\Omega)[1+i(\Omega)] \text { for all } \omega,
\end{aligned}
$$

where demand in each state satisfies the buyer's first-order condition for consumption; i.e., it satisfies the preceding constraints. The first-order condition for $p$ yields the pricing equation

$$
p\left(\Omega_{-1}\right)=\frac{\int_{\Omega} \sigma[s(\Omega)] s(\Omega) q(\Omega)(1 / \alpha) c^{\prime}[q(\Omega)] d F\left(\omega \mid \Omega_{-1}\right)}{\left(1-R^{u}\right) \int_{\Omega} \phi(\Omega) \sigma[s(\Omega)] s(\Omega) q(\Omega) d F\left(\omega \mid \Omega_{-1}\right)},
$$

where we have taken into account that in a symmetric equilibrium $\pi(\Omega)=$ $s(\Omega) / \mathbf{N}$. Equation (29) then replaces $p$ in (23). We can then combine (20) and (29) to get an expression for the interest rate,

$$
1+i(\Omega)
$$

$$
=\frac{\left(1-R^{u}\right) \varepsilon u^{\prime}\{\sigma[s(\Omega)] s(\Omega) q(\Omega)\} \int_{\Omega} \phi(\Omega) \sigma[s(\Omega)] s(\Omega) q(\Omega) d F\left(\omega \mid \Omega_{-1}\right)}{\phi(\Omega) \int_{\Omega} \sigma[s(\Omega)] s(\Omega) q(\Omega)(1 / \alpha) c^{\prime}[q(\Omega)] d F\left(\omega \mid \Omega_{-1}\right)} .
$$




\section{OPTIMAL STABILIZATION}

We now derive the optimal stabilization policy in symmetric stationary monetary equilibrium. To study this problem, we pursue the primal approach to the Ramsey problem, where the central bank chooses the quantities $x_{t}, y_{t}, q_{t}^{b}, q_{t}, s_{t}$ to maximize (3) subject to the free entry condition (18), the relevant pricing protocol, and the resource constraints. In the Appendix, we show that these quantities can be implemented with history-dependent injections $\tau_{1}(\Omega)$ and $\tau_{3}(\Omega)$ that satisfy $i_{t} \geq 0$ and (23). With competitive pricing the pricing protocol is (25), with statecontingent monopoly pricing it is (27), and with price posting it is (29). It should be clear that in all cases $x_{t}=x^{*}$ and $y_{t}$ is determined by the household's budget constraint once all of the other quantities are chosen. Finally, from (24), $q_{t}^{b}$ is determined once we have $q_{t}$ and $s_{t}$. So the central bank's problem reduces to choosing $q_{t}$ and $s_{t}$.

PROPOSITION 1. Consider the case of competitive pricing. The constrained optimal allocation is stationary and depends only on the current state $\omega$. When $\sigma^{\prime}[s(\omega)]=0, i(\omega)=0, q(\omega)=q^{*}(\omega)$, and $s(\omega)=s^{*}(\omega)$ for all states. When $\sigma^{\prime}[s(\omega)]<0, i(\omega)>0, q(\omega)<q^{*}(\omega)$, and $s(\omega)>s^{*}(\omega)$ for all states.

The allocation is stationary and depends only on the current state in both cases, despite the persistence of the shocks. The reason is that the only equation for which the persistence of the shocks matters is the money demand equation, (23). Given its optimal choices $\{q(\omega), s(\omega)\}_{\omega \in \Phi}$, the central bank then chooses $\gamma(\omega)$ to ensure (23). Thus, any information content provided by the persistence of the shocks is offset by choosing the stochastic inflation rate appropriately.

With $\sigma^{\prime}[s(\omega)]=0$, the Friedman rule replicates the first-best allocation. This can be seen by noting that from (26), when $i(\omega)=0, q(\omega)=q^{*}(\omega)$. In the proof of Proposition 1, we show that the zero-profit condition that determines entry satisfies

$$
k(s)=\left[c^{\prime}(q) q-c(q)\right] / \alpha_{t} .
$$

Thus, with $\sigma^{\prime}[s(\omega)]=0$, the entry condition for competitive pricing (31) replicates (5) at $q(\omega)=q^{*}(\omega)$. The intuition is that if $\sigma^{\prime}[s(\omega)]=0$, there is no congestion externality, and the only friction is the cost of holding money across periods. Under the Friedman rule the agents are compensated for these costs and so agents perfectly self-insure against all shocks. Consequently, there are no welfare gains from stabilization policies. Note that Proposition 1 also holds in a model where the number of sellers is exogenously given.

With $\sigma^{\prime}[s(\omega)]<0$, the central bank never chooses $i(\omega)=0$. The reason is the congestion externality. Sellers ignore how their entry lowers the expected profits of other sellers. Consequently, at equilibrium there are too many sellers and the aggregate entry cost $s(\omega) \kappa$ is too high relative to the social optimum. To see why a deviation from the Friedman rule is optimal, assume that the central bank sets $i(\omega)=0$, which generates the efficient quantity $q(\omega)=q^{*}(\omega)$ in all states. Now consider a reduction in $s(\omega)$ when $i(\omega)=0$. When $s(\omega)$ is marginally reduced, 
$q(\omega)$ is also marginally reduced, but the first-order welfare loss from doing so is zero. The reduction in $q(\omega)$ reduces expected profits for sellers, and thus entry declines. This produces a first-order gain in welfare from reducing $s(\omega) \kappa$. This is achieved by increasing $i(\omega)$ above zero.

Although the preceding argument does not require $i(\omega)>0$ for all states, nevertheless it is optimal to have this. The reason is that the central bank wants to smooth consumption across states. Intuitively, consider two states $\omega, \omega^{\prime} \in \Phi$ with $i(\omega)=0$ implying $q(\omega)=q^{*}(\omega)$, and $i\left(\omega^{\prime}\right)>0$ implying $q\left(\omega^{\prime}\right)<q^{*}\left(\omega^{\prime}\right)$. Then the first-order loss from decreasing $q(\omega)$ is zero, whereas there is a first-order gain from increasing $q\left(\omega^{\prime}\right)$. This gain can be accomplished by increasing $i(\omega)$ and lowering $i\left(\omega^{\prime}\right)$. Thus, the central bank's optimal policy is to set $i(\omega)>0$ for all states.

We find this result interesting because it is very reminiscent of the view that the Federal Reserve kept interest rates "too low for too long" from 2003 to 2005. In short, this argument implies that the Fed should have raised interest rates to choke off entry into the housing and commercial property markets. It is important to note that this argument requires some type of congestion externality to make entry inefficient. Entry per se is not enough.

Last, with the central bank's optimal interest rate policy, $\operatorname{di}(\omega) / d q(\omega)$ has the same sign as $d R^{c}(\omega) / d q(\omega)$, where $R^{c}$ is the elasticity of the marginal cost function. Thus, if the elasticity of the marginal cost function is constant, we have $d i(\omega) / d q(\omega)=0$, and the central bank smooths interest rates perfectly. The central bank moves the nominal interest rate in a countercyclical fashion when $d R^{c}(\omega) / d q(\omega)>0$ and in a procyclical manner when the opposite is true.

\section{Example 2}

Using our assumed functional forms, the central bank's optimal allocation is given by

$$
\begin{aligned}
q^{c}(\omega) & =\left(\frac{\theta+\rho}{\theta \rho+\rho}\right)^{\theta \delta}\left(\frac{1}{1+\theta}\right)^{\eta \delta} q^{*}(\omega)<q^{*}(\omega), \\
s^{c}(\omega) & =\left(\frac{1}{1+\theta}\right)^{(1-\eta) \delta}\left(\frac{\theta+\rho}{\rho}\right)^{\rho \delta} s^{*}(\omega)>s^{*}(\omega), \\
i^{c}(\omega) & =\frac{\theta(\rho-1)}{\theta+\rho}>0 .
\end{aligned}
$$

This example illustrates the basic insight of the model. When entry is endogenous, too much entry occurs. To reduce entry, the central bank inflates in order to drive up nominal interest rates. This lowers consumption of market 2 goods and lowers profits for sellers. Expected lower profits reduce entry by sellers. Because $R^{c}(\omega)=\rho-1$ for these functional forms, the optimal nominal interest rate is constant across states. Note also that when there are no search externalities-i.e., when $\theta=0$ - then $q^{c}(\omega)=q^{*}(\omega), s^{c}=s^{*}$, and $i^{c}(\omega)=0$. Again, the central bank wants entry to be procyclical. 
We next consider the case of state-contingent monopoly pricing.

PROPOSITION 2. Consider the case of state-contingent monopoly pricing. The constrained optimal allocation is stationary and depends only on the current state $\omega$. When $\sigma^{\prime}(s)=0, i(\omega)=0, q(\omega)<q^{*}(\omega)$, and $s(\omega)>s^{*}(\omega)$ for all states. When $\sigma^{\prime}(s)<0, i(\omega)>0, q(\omega)<q^{*}(\omega)$, and $s(\omega)>s^{*}(\omega)$ for all states.

With monopoly pricing and $\sigma^{\prime}(s)=0$, the Friedman rule is again optimal. However, the first-best allocation cannot be achieved because the monopoly pricing distortion causes $q(\omega)$ to be inefficiently low and $s(\omega)$ to be inefficiently high in all states.

With endogenous entry, once again, because of the entry externality, the central bank pushes up interest rates to reduce profits and thus entry. As with competitive pricing, entry is higher than the social optimum. Also, production is lower than $q^{*}$ and $q^{c}$.

Finally, we study the case of non-state-contingent monopoly pricing.

PROPOSITION 3. Consider the case of price posting. In this case, the central bank replicates the optimal allocation that occurs under state-contingent monopoly pricing.

Why is the central bank able to replicate the posting allocation? Posting simply imposes a constraint on the behavior of $p$ in market 2 . However, the central bank only cares about the relative price $\phi(\omega) p(\omega)$ between market 2 and market 3 . As long as that is flexible, the central bank can replicate the state-contingent monopoly price allocation.

\subsection{Discussion}

We first compare our results with those of Ireland (1996), because our paper is most closely related to his article. Ireland (1996) studies optimal stabilization policies when firms set nominal prices one period in advance, which corresponds to price posting in our model. He finds that when the economy is subject to aggregate demand shocks, the optimal policy is to make the average money growth rate small. Under this policy, there is no need to respond actively to these shocks. He also reports that one class of optimal policies is the constant money growth rate $\gamma=\beta$ advocated by Friedman (1969). Under this policy, the opportunity cost of holding money is zero and so agents are willing to hold a stock of real balances that is large enough to offset any demand shocks. ${ }^{19}$ In the absence of a congestion externality, we find the same result for all three pricing protocols. In this case, the optimal policy is the Friedman rule; i.e., to set $i(\omega)=0$.

Khan et al. (2003) study a monetary economy with imperfect competition and sticky prices. They also find that with fully flexible prices the Friedman rule is optimal but cannot achieve the first-best allocation because of monopolistic distortion in the price setting. With costly price setting, the optimal nominal interest rate is positive. Furthermore, they find that in response to real and nominal aggregate 
shocks, the optimal policy is to stabilize the price level around a deflationary trend path.

The key difference between our approach and the New Keynesian literatureas represented by Khan et al. (2003) - is that these models have staggered price stickiness, which results in a nondegenerate distribution of prices. This prevents the central bank from getting the relative price between flexible and sticky prices "right." The reason is that there are many relative prices that are out of alignment, whereas we only have one. To address this inefficiency the central bank essentially stabilizes the price level and lets output move.

In our model, the optimal policy requires inflation to be stochastic so that the price level moves around. Expected inflation is what changes the real value of money and thus adjusts the liquidity constraints. This result is very different from what one obtains in New Keynesian models. In those models, inflation distorts relative prices because of stickiness. So, to keep output close to the efficient level, the central bank strongly stabilizes the price level to the point that it looks like price level targeting. However, in our paper, the reverse is true-we optimally choose stochastic inflation to move allocations closer to the efficient level. The implementation scheme and associated stochastic inflation rate that yields the optimal allocation is characterized in the following.

\subsection{Implementation}

In the Appendix, we derive implementation schemes for each pricing protocol that supports the desired allocation when $\sigma^{\prime}[s(\omega)]<0$. The schemes are not unique, because the transfers are nominal injections and the central bank cares only about the relative transfers across states.

The key thing to note is that the inflation rate is state-dependent and serially correlated. The reason for the serial correlation is as follows. The optimal allocation associated with state $\omega$ does not depend on $\Omega_{-1}$. However, $\Omega_{-1}$ contains information about the future state $\omega$, and this affects agents' demand for real balances at time $t-1$, as is shown by (23). To offset any informational value that history has in relation to current money demand, the central bank offers a menu of state-contingent transfers that makes the real value of money constant regardless of $\Omega_{-1}$.

Note that for general shock processes, the central bank must promise a sequence of transfers for all possible histories. This seems to be an unrealistic implementation policy in practice. However, if the shocks are Markovian, then the central bank's transfers only need to be conditioned on the current and previous state-a much simpler set of transfers to implement.

\section{CONCLUSION}

In this paper, we construct a DSGE model where money is essential for trade and where a search externality creates "congestion," which causes aggregate output to be inefficient. We introduce a variety of well-defined aggregate shocks that 
generate consumption risk for households. Because of the informational frictions that give rise to money, households are unable to insure themselves perfectly against these shocks. This gives rise to a welfare-improving role for monetary policy that works by adjusting the nominal interest rate in response to these shocks.

In this setting, our basic results concerning the optimal stabilization policy are as follows. With a fixed probability of trading, the optimal monetary policy is to run the Friedman rule and set the nominal interest rate to zero in all states. This is true for all three pricing protocols. When the trading probability depends on aggregate entry, a congestion externality arises that makes entry inefficiently high. Thus, the central bank finds it optimal to raise interest rates above zero in all states in order to reduce profits and deter entry even though it lowers average consumption. Once again, this is true for all pricing protocols. In short, the zero lower bound is never a binding constraint in our model. The key to implementing the desired allocation is to manipulate the relative price of goods across markets by choosing state-dependent nominal interest rates.

There are many extensions of this model that would be interesting to pursue. For example, how would the optimal policy be affected if the repayment of loans were endogenous? In particular, does the risk of default alter stabilization? Given the events of 2007 to 2009 , the role of default on stabilization policy appears to be an important issue. Furthermore, we assume that the shocks are known to the central bank. An interesting question is what is the optimal policy if the central bank has imperfect information about the nature of the aggregate shocks? How would the existence of inside money affect the equilibrium and optimal policy? For example, would inside money act as an automatic stabilizer, eliminating the need for the central bank to stabilize the economy?

Finally, one could also use our framework to address the classical question raised in Lucas (1987): how much is the welfare gain associated with eliminating the business cycle? In the context of our model, this would mean calculating the benefits of applying the optimal stabilization policy versus a passive policy, where the central bank would not intervene in response to aggregate productivity and preference shocks. Lucas (1987) and the following literature, surveyed in Barlevy (2005), found that the welfare benefits of eliminating the business cycle are surprisingly small. An interesting research question would be to see whether a microfounded framework with search externalities would generate larger benefits.

\section{NOTES}

1. Most stabilization policy analysis has been done using the canonical New Keynesian model with sticky prices. In this model, in the absence of nominal rigidities, monetary policy is ineffective because money is neutral. An important contribution of our paper is to show that the informational frictions that give rise to a medium-of-exchange role for money allow a welfare-improving role of stabilization policy even when all prices are fully flexible. 
2. Rocheteau and Wright (2009) is a quantitative version of Rocheteau and Wright (2005), where the extensive margin is modeled as an agent's endogenous choice of whether to be a buyer or a seller. They find that a small deviation from the Friedman rule can improve welfare if there are too many buyers. When inflation increases, the cost of holding money increases and agents prefer to become sellers. As long as this effect is not outweighed by the negative effect on the intensive margin, i.e., quantity per trade, a deviation from the Friedman rule can increase the number of sellers and, hence, the number of trades at equilibrium.

3. In our model, for all pricing protocols, the only externality is related to the entry congestion. There is neither a consumer surplus effect (through increased product diversity) nor a profit destruction effect (through a reduction in the price level) as in Bilbiie et al. (2008).

4. The environment combines elements of Lagos and Wright (2005) and Berentsen et al. (2007). The Lagos-Wright framework provides a microfoundation for money demand while keeping the distribution of money balances analytically tractable. Berentsen et al. (2007) introduce financial intermediation into the Lagos-Wright framework.

5. By "essential" we mean that the use of money enlarges the set of incentive-feasible allocations.

6. As in Lagos and Wright (2005), these assumptions allow us to obtain a degenerate distribution of money holdings at the beginning of a period. The different utility functions $v(\cdot)$ and $u(\cdot)$ allow us to impose technical conditions such that at equilibrium all agents produce and consume in the last market.

7. This restriction on preferences is not necessary for competitive pricing but is needed for interior solutions under monopolistic pricing.

8. We have also allowed this number to be different from $1 / 2$ and to be made random. However, doing so has added very little to the analytical and quantitative results. Thus, we chose $1 / 2$ to simplify notation.

9. In Berentsen et al. (2007) we derive the equilibrium under the assumption that the only punishment for strategic default is exclusion from the financial system in all future periods.

10. Assuming a fixed utility cost $\kappa$ is standard in the labor search literature [e.g., Pissarides (2000), Rogerson et al. (2005)] or in the money search literature [e.g., Rocheteau and Wright (2005)]. Note that exit is exogenous and constant in the model, because the entry cost has to be paid every period, which implies that the firm exit rate is equal to $100 \%$.

11. See our discussion of the literature in the Introduction.

12. Note that because the cash injections happen after the demand shock is realized, the central bank could give the injections to the buyers only. Nevertheless, one can show that such targeted injections do not affect the qualitative results of our model. Furthermore, untargeted lump-sum injections do not require that the central bank observe the state of the individual household (buyer or nonbuyer).

13. If the productivity shock affects the entry cost in the same way as the cost function, then $s^{*}(\omega)$ is increasing in $\alpha$ as well.

14. The MRS between the two markets is $\varepsilon u^{\prime}\left[q^{b}(\omega)\right] / v^{\prime}[x(\omega)]$. But from the optimization problem in market $3, v^{\prime}[x(\omega)]=1$ for all $\omega$.

15. This simply requires that the measure of potential sellers be sufficiently large so that there is no state for which all of them want to enter. This is a standard assumption in the labor search literature.

16. This assumption is made simply to compare the allocation with monopoly pricing with one with competitive pricing. For this reason, we ignore issues involving $1<\sigma[s(\Omega)] s(\Omega)$.

17. Given our simple approach to generating monopoly power, the gross markup is given by $\left(1-R^{u}\right)^{-1}$, which is constant. Changing the matching function so that the markup depends negatively on entry, as in Jaimovich and Floettoto (2008), would be an interesting extension.

18. For this case, we have in mind restaurants that print their menus in advance but upon seeing the state of the economy can choose to open or not. 
19. In contrast, for aggregate supply shocks, he finds that the optimal policy requires the money supply to respond actively and that the optimal policy is procyclical.

\section{REFERENCES}

Aruoba, S.B. and S.K. Chugh (2010) Optimal fiscal and monetary policy when money is essential. Journal of Economic Theory 145, 1618-1647.

Aruoba, S.B., G. Rocheteau, and C. Waller (2007) Bargaining and the value of money. Journal of Monetary Economics 54, 2636-2655.

Barlevy, G. (2005) The cost of business cycles and the benefits of stabilization. Economic Perspectives, Federal Reserve Bank of Chicago 29, 32-49.

Berentsen, A., G. Camera, and C. Waller (2007) Money, credit and banking. Journal of Economic Theory 135, 171-195.

Berentsen, A., G. Rocheteau, and S. Shi (2007) Friedman meets Hosios: Efficiency in search models of money. Economic Journal 117, 174-195.

Berentsen, A. and C. Waller (2011) Price level targeting and optimal stabilization. Journal of Money, Credit and Banking 43(7), 559-580.

Bergin, P. and G. Corsetti (2008) The extensive margin and monetary policy. Journal of Monetary Economics 55, 1222-1237.

Bilbiie, F., F. Ghironi, and M. Melitz (2007). Monetary policy and business cycles with endogenous entry and product variety. NBER Macro Annual 2007, 299-354.

Bilbiie, F., F. Ghironi, and M. Melitz (2008). Monopoly Power and Endogenous Variety: Distortions and Remedies. NBER working paper 14383.

Correia, I., J.P. Nicolini, and P. Teles (2008) Optimal fiscal and monetary policy: Equivalence results. Journal of Political Economy 116(1), 141-170.

Ireland, P. (1996) The role of countercyclical monetary policy. Journal of Political Economy 104 vol. 4, 704-723.

Jaimovich, N. and M. Floetotto (2008) Firm dynamics, markup variations, and the business cycle. Journal of Monetary Economics 55(7), 1238-1252.

Khan, A., R. King, and A. Wolman (2003) Optimal monetary policy. Review of Economic Studies 70(4), 825-860.

Lagos, R. and G. Rocheteau (2005) Inflation, output and welfare. International Economic Review46, 495-522.

Lagos, R. and R. Wright (2005) A unified framework for monetary theory and policy evaluation. Journal of Political Economy 113, 463-484.

Lewis, V. (2009) Optimal Monetary Policy and Firm Entry. NBB working paper 178, October.

Liu, L., L. Wang, and R. Wright (2011) On the "hot potato" effect of inflation: Intensive versus extensive margins. Macroeconomic Dynamics 15(S2), 191-216.

Lucas, R. (1987) Models of Business Cycles. Oxford, UK: Basil Blackwell.

Pissarides, C. (2000) Equilibrium Unemployment Theory, 2nd ed. Cambridge, MA: MIT Press.

Rocheteau, G. and R. Wright (2005). Money in search equilibrium, in competitive equilibrium and in competitive search equilibrium. Econometrica 73, 75-202.

Rocheteau, G. and R. Wright (2009) Inflation and welfare in models with trading frictions. In David E. Altig and Ed Nosal (eds.), Monetary Policy in Low Inflation Economies, pp. 89-116. Cambridge University Press.

Rogerson, R., R, Shimer, and R. Wright (2005) Search-theoretic models of the labor market: A survey. Journal of Economic Literature 43, 959-988.

Shi, S. (1997) A divisible search model of fiat money. Econometrica 65, 75-102. 


\section{APPENDIX}

\section{A.1. FIRST-BEST ALLOCATION}

The planner chooses the allocation

$$
A_{t} \equiv\left[q_{t}^{b}(j)_{j \in \mathcal{B}}, q_{t}(j)_{j \in \tilde{\mathcal{S}}}, x_{t}(j)_{j \in \mathcal{H} \cup \mathcal{F}}, y_{t}(j)_{j \in \mathcal{H} \cup \mathcal{F}}\right]
$$

and the measures of sellers $s_{t}$ who enter the market for each period. The quantities $q_{t}^{b}(j)_{j \in \mathcal{B}}$ are the consumption quantities of all households with $e=\varepsilon$ (the buyers), and $q_{t}(j)_{j \in \tilde{\mathcal{S}}}$ are the production quantities of all sellers that pay the entry cost and are able to enter market 2 , whereas $x_{t}(j)_{j \in \mathcal{H} \cup \mathcal{F}}$ are the consumption quantities of all households and all sellers in

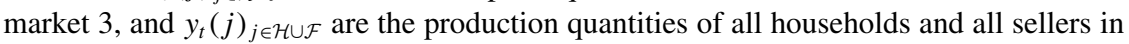
market 3 . The planner is constrained that the allocation has to be feasible. In the second and third markets, respectively, for each state $\omega \in \Omega$ and each date $t$, this requires that

$$
\begin{aligned}
\int_{\mathcal{B}} q_{t}^{b}(j) d j & \leq \int_{\tilde{\mathcal{S}}} q_{t}(j) d j, \\
\int_{\mathcal{H} \cup \mathcal{F}} x_{t}(j) d j & \leq \int_{\mathcal{H} \cup \mathcal{F}} y_{t}(j) d j .
\end{aligned}
$$

An efficient allocation is defined as paths for

$$
q_{t}^{b}(j)_{j \in \mathcal{B}}, q_{t}(j)_{j \in \tilde{\mathcal{S}}}, x_{t}(j)_{j \in \mathcal{H} \cup \mathcal{F}}, y_{t}(j)_{j \in \mathcal{H} \cup \mathcal{F}} \text {, and } s_{t}
$$

that maximize (3) subject to (A.1) and (A.2) and an initial aggregate state $\omega_{0}$. One can easily show that it is optimal to treat all agents of the same type equally. Moreover, using (A.1), it is straightforward to show that the planner allocation yields

$$
\int_{\Phi} \int_{\mathcal{H} \cup \mathcal{F}}\left[v\left(x_{t}\right)-y_{t}\right] d F\left(\omega_{t} \mid \Omega_{t-1}\right)=\int_{\mathcal{H} \cup \mathcal{F}} v\left(x^{*}\right)-x^{*},
$$

which is not state-contingent, so we can ignore this term in (3). Accordingly, the Lagrangian of the planner problem is

$$
\begin{gathered}
L_{p}=\int_{\Phi}\left[\varepsilon_{t} u\left(q_{t}^{b}\right)-\sigma\left(s_{t}\right) s_{t} c\left(q_{t}\right) / \alpha_{t}\right] d F\left(\omega_{t} \mid \Omega_{t-1}\right) \\
-\int_{\Phi} s_{t} \kappa d F\left(\omega_{t} \mid \Omega_{t-1}\right)+\mu_{t}\left[\sigma\left(s_{t}\right) s_{t} q_{t}-n_{t} q_{t}^{b}\right] .
\end{gathered}
$$

The FOCs for this problem after simplification are

$$
\begin{aligned}
& 0=\varepsilon_{t} u^{\prime}\left(q_{t}^{b}\right)-\mu_{t}, \\
& 0=\mu_{t}-c\left(q_{t}\right) / \alpha_{t}, \\
& 0=\sigma\left(s_{t}\right)\left(1+\Sigma^{\sigma}\right)\left[c^{\prime}\left(q_{t}\right) q_{t}-c\left(q_{t}\right)\right] / \alpha_{t}-\kappa, \\
& 0=\sigma\left(s_{t}\right) s_{t} q_{t}-q_{t}^{b},
\end{aligned}
$$

for all $t$. It is clear from these FOCs that the optimal allocation is independent of $\Omega_{t-1}$ and stationary for all $\omega \in \Phi$, implying that, for a given state $\omega, x_{t}=x(\omega), y_{t}=y(\omega)$, 
$q_{t}^{b}=q^{b}(\omega), q_{t}=q(\omega)$ for all $t$. Furthermore, an interior solution for $s_{t}$ requires $-\Sigma^{\sigma}<1$, which we have assumed.

Define $k[s(\omega)] \equiv \kappa / \sigma[s(\omega)]$ with $k^{\prime}(s)>0$. To prove existence and uniqueness of the first-best allocation, we can rearrange (4)-(5) as follows:

$$
\begin{array}{r}
\frac{\varepsilon u^{\prime}\{\sigma[s(\omega)] s(\omega) q(\omega)\}}{c^{\prime}[q(\omega)] / \alpha}-1=0, \\
k[s(\omega)]-\left(1+\Sigma^{\sigma}\right)\left\{c^{\prime}[q(\omega)] q(\omega)-c[q(\omega)]\right\} / \alpha=0 .
\end{array}
$$

(A.3) is a strictly decreasing function in $[s(\omega), q(\omega)]$ space. It approaches infinity as $s(\omega)$ approaches zero and approaches zero as $s(\omega)$ goes to infinity. If $k^{\prime}[s(\omega)]>0$, (A.4) is strictly increasing in $[s(\omega), q(\omega)]$ space. Moreover, at $s(\omega)=0$, there exists a finite $q(\omega)>0$ that solves

$$
k[s(\omega)]-\left(1+\Sigma^{\sigma}\right)(1 / \alpha)\left\{c^{\prime}[q(\omega)] q(\omega)-c[q(\omega)]\right\}=0 .
$$

Hence, a unique solution $\left[s^{*}(\omega), q^{*}(\omega)\right]$ exists. If $\sigma[s(\omega)]=\sigma$, (A.4) is independent of $s(\omega)$ implying that for $\kappa<+\infty$, a unique solution $\left[s^{*}(\omega), q^{*}(\omega)\right]$ exists.

Proof of Proposition 1. The proof involves three steps. We first derive the solution to the central bank's problem. We then demonstrate that the solution satisfies $i\left(\omega_{t}\right) \geq 0$. Finally, we show that there exists a transfer scheme $\tau_{1}(\Omega)$ and $\tau_{3}(\Omega)$ that implements the central bank's allocation for each $\Omega$ and satisfies (23).

First step. The central bank allocation has to satisfy two constraints. The first constraint is the entry condition (18), which holds in each state. The second constraint is the pricing equation (25), which also holds in each state. We can use (18) to eliminate $p_{t}\left(\Omega_{t}\right) \phi_{t}\left(\Omega_{t}\right)$ from (25) to get

$$
k(s)=\left[c^{\prime}\left(q_{t}\right) q_{t}-c\left(q_{t}\right)\right] / \alpha_{t} .
$$

The central bank then maximizes (3) subject to (A.5). Using (A.2), it is straightforward to show that the optimal policy yields

$$
\int_{\Phi} \int_{\mathcal{H} \cup \mathcal{F}}\left[v\left(x_{t}\right)-y_{t}\right] d F\left(\omega_{t} \mid \Omega_{t-1}\right)=\int_{\mathcal{H} \cup \mathcal{F}} v\left(x^{*}\right)-x^{*},
$$

which is not state-contingent or dependent on monetary policy, so we can ignore this term in (3). Consequently, using (24), the central bank's problem reduces to

$$
\begin{aligned}
\mathcal{V} & =\sum_{t=0}^{\infty} \beta^{t} \int_{\Phi}\left[\varepsilon_{t} u\left(q_{t}^{b}\right)-\sigma\left(s_{t}\right) s_{t} c\left(q_{t}\right) / \alpha_{t}\right] d F\left(\omega_{t} \mid \Omega_{t-1}\right) \\
& -\sum_{t=0}^{\infty} \beta^{t} \int_{\Phi} \sigma\left(s_{t}\right) s_{t} k\left(s_{t}\right) d F\left(\omega_{t} \mid \Omega_{t-1}\right) .
\end{aligned}
$$


The Lagrangian is

$$
\begin{aligned}
L_{c} & =\sum_{t=0}^{\infty} \beta^{t} \int_{\Phi}\left\{\varepsilon_{t} u\left(q_{t}^{b}\right)-\sigma\left(s_{t}\right) s_{t} c\left(q_{t}\right) / \alpha_{t}\right\} d F\left(\omega_{t} \mid \Omega_{t-1}\right) \\
& -\sum_{t=0}^{\infty} \beta^{t} \int_{\Phi} \sigma\left(s_{t}\right) s_{t} k\left(s_{t}\right) d F\left(\omega_{t} \mid \Omega_{t-1}\right) \\
& +\sum_{t=0}^{\infty} \beta^{t} \int_{\Phi} \mu_{t}\left[\sigma\left(s_{t}\right) s_{t} q_{t}-q_{t}^{b}\right] d F\left(\omega_{t} \mid \Omega_{t-1}\right) \\
& +\sum_{t=0}^{\infty} \beta^{t} \int_{\Phi} \hat{\lambda}_{t}\left\{k\left(s_{t}\right)-\left[c^{\prime}\left(q_{t}\right) q_{t}-c\left(q_{t}\right)\right] / \alpha_{t}\right\} d F\left(\omega_{t} \mid \Omega_{t-1}\right),
\end{aligned}
$$

where $\mu_{t}$ and $\hat{\lambda}_{t} \equiv s_{t} \sigma\left(s_{t}\right) \lambda_{t}$ is the time- $t$ Lagrangian multiplier for state $\omega_{t}$. Then for all $t$ the central bank's allocation satisfies

$$
\begin{aligned}
& 0=\varepsilon_{t} u^{\prime}\left(q_{t}^{b}\right)-\left(1+\lambda_{t} R^{c}\right) c^{\prime}\left(q_{t}\right) / \alpha_{t}, \\
& 0=\varepsilon_{t} u^{\prime}\left(q_{t}^{b}\right) q_{t}-c\left(q_{t}\right) / \alpha_{t}-k\left(s_{t}\right)-\left(1+\Sigma^{\sigma}\right)^{-1}\left(1-\lambda_{t}\right) s_{t} k^{\prime}\left(s_{t}\right), \\
& 0=k\left(s_{t}\right)-\left[c^{\prime}\left(q_{t}\right) q_{t}-c\left(q_{t}\right)\right] / \alpha_{t}, \\
& 0=q_{t}^{b}-\sigma\left(s_{t}\right) s_{t} q_{t},
\end{aligned}
$$

where (A.6) and (A.7) are the FOCs for $q_{t}$ and $s_{t}$, respectively. Note that there are no terms involving past or future values in (A.6)-(A.8), so the allocation is stationary. Hence, as with the planner, the central bank faces no intertemporal trade-offs, and so for each aggregate state $\omega$ we have $q_{t}=q(\omega)$ and $s_{t}=s(\omega)$. For notational convenience, we now drop the dependence of $q$ and $s$ on $\omega$, with the understanding that they are state-dependent.

Use (A.6) and (A.8) to write (A.7) as follows

$$
\lambda=\frac{s k^{\prime}(s)}{R^{c}\left(1+\Sigma^{\sigma}\right) c^{\prime}(q) q / \alpha+s k^{\prime}(s)} .
$$

Note that $\lambda<1$. When (A.10) is used to replace $\lambda$ in (A.6), $q$ and $s$ satisfy

$$
\begin{aligned}
\frac{\varepsilon u^{\prime}[\sigma(s) s q]}{(1 / \alpha) c^{\prime}(q)}-1 & =\frac{R^{c} s k^{\prime}(s)}{R^{c}\left(1+\Sigma^{\sigma}\right)(1 / \alpha) c^{\prime}(q) q+s k^{\prime}(s)}, \\
k(s) & =\left[c^{\prime}(q) q-c(q)\right] / \alpha .
\end{aligned}
$$

In $(s, q)$ space, (A.11) approaches infinity as $s$ approaches zero and it approaches zero as $s$ goes to infinity. If $k^{\prime}(s)>0,\left(\right.$ A.12) is strictly increasing and $q \geq 0$ solves $k-\left[c^{\prime}(q) q-\right.$ $c(q)] / \alpha=0$ at $s=0$. Hence, a solution $(s, q)$ exists. If $k^{\prime}(s)=0$, then $k(s)=k$ and (A.12) is independent of $s$, implying that for $k<+\infty$, a solution $(s, q)$ exists. If (A.11) is strictly decreasing in $(q, s)$ space, the equilibrium is unique.

By comparing these two expressions to the first-best allocation (A.3)-(A.4), it is straightforward to show that $q<q^{*}$. 
We now prove that $s \geq s^{*}$. Suppose that the central bank is constrained to implement $s^{*}$. Then $q$ solves

$$
k\left(s^{*}\right)-\left[c^{\prime}(q) q-c(q)\right] / \alpha=0 .
$$

Let $q^{c}$ denote the value of $q$ that solves (A.13) and let $s^{c} \equiv s^{*}$. From (A.4), it is clear that $q^{c}<q^{*}$. Now let the central bank choose $q$ and $s$. Assume-contrary to the claim in the Proposition-that the optimal allocation satisfies $s<s^{c} \equiv s^{*}$. Then, from (A.13), $q<q^{c}$. It is evident that the allocation $(q, s)$ has lower welfare than $\left(q^{c}, s^{c}\right)$, because $q<q^{c}<q^{*}$ and $s<s^{c}=s^{*}$. Thus, in any competitive equilibrium, $s \geq s^{*}$.

Second step. From (26) we have $1+i=\frac{\varepsilon u^{\prime}[\sigma(s) s q]}{c^{\prime}(q) / \alpha}$. Hence, using (A.11) yields

$$
i=\frac{R^{c} s k^{\prime}(s)}{R^{c}\left(1+\Sigma^{\sigma}\right) c^{\prime}(q) q / \alpha+s k^{\prime}(s)} .
$$

Consequently, if $k^{\prime}(s)>0, i>0$ in all states. If $k^{\prime}(s)=0, i(\omega)=0$ in all states. Note that if $s k^{\prime}(s) / k(s)=\chi$ and $q c^{\prime}(q) / c(q)=v>1$ are constants, then $R^{c}=v-1$ and the free entry condition reduces to $k(s)=(v-1) c(q) / \alpha$. It then follows that $i$ is constant and given by

$$
i=\frac{\chi(v-1)}{\left(1+\Sigma^{\sigma}\right) v+\chi}
$$

Third step. We now show that a set of transfers $\tau_{1}(\Omega)$ and $\tau_{3}(\omega)$ exists that implement the central bank's allocation and satisfy (23). Using (24) and (25), we can write (23) as follows:

$$
1=\beta \int_{\Phi}\left\{\frac{\alpha \varepsilon u^{\prime}\{\sigma[s(\omega)] s(\omega) q(\omega)\}}{\gamma(\Omega) c^{\prime}[q(\omega)]}\right\} d F\left(\omega \mid \Omega_{t-1}\right) .
$$

We first consider the case $k^{\prime}(s)=0$. In this case, (A.14) reduces to

$$
1=\beta \int_{\Phi} \frac{1}{\gamma(\Omega)} d F\left(\omega \mid \Omega_{t-1}\right)=\beta \int_{\Phi} \frac{1}{1+\tau_{1}(\Omega)+\tau_{3}(\Omega)} d F\left(\omega \mid \Omega_{t-1}\right) .
$$

It is clear that any set of transfers $\tau_{1}(\Omega)$ and $\tau_{3}(\Omega)$ that satisfies $\gamma(\Omega)=1+\tau_{1}(\Omega)+\tau_{3}(\Omega)=$ $\beta$ for all $\Omega$ implements the central bank's allocation.

Consider next the case $k^{\prime}(s)>0$. Assume that the transfers are such that the agents have just enough money to buy $q^{b}(\omega)=\sigma[s(\omega)] s(\omega) q(\omega)$ in each state; i.e., $p(\Omega) q^{b}(\omega)=$ $M_{-1}\left[1+\tau_{1}(\Omega)\right]$. From the pricing equation (25) we can write this expression as follows:

$$
q^{b}(\omega) c^{\prime}[q(\omega)] / \alpha=\phi(\Omega) M_{-1}\left[1+\tau_{1}(\Omega)\right] .
$$

Let $z \equiv \phi(\Omega) M(\Omega)=\phi(\Omega) M_{-1}\left[1+\tau_{1}(\Omega)+\tau_{3}(\Omega)\right]$. Using (A.15) we get

$$
z=q^{b}(\omega) c^{\prime}[q(\omega)] / \alpha+\phi(\Omega) M_{-1} \tau_{3}(\Omega)
$$

We have one degree of freedom for the choice of $\tau_{3}(\Omega)$. Assume the central bank conditions the transfers on the $t$ and $t-1$ shocks for any $\Omega_{-2}$. We then have $\tau_{3}(\Omega)=\tau_{3}\left(\omega, \omega_{-1}, \Omega_{-2}\right)$. Consider the state $\omega_{L}=\left(\alpha_{L}, \varepsilon_{L}\right)$ and set $\tau_{3}\left(\omega_{L}, \omega_{L}, \Omega_{-2}\right)=0$. This pins down the real stock of money $z=q^{b}\left(\omega_{L}\right) c^{\prime}\left[q\left(\omega_{L}\right)\right] / \alpha_{L}$. This implies that $\gamma\left(\omega_{L}, \omega_{L}, \Omega_{-2}\right)=1+$ $\tau_{1}\left(\omega_{L}, \omega_{L}, \Omega_{-2}\right)$, which remains to be determined. We will return to this later. Now, using the value of $z$ in (A.16) for $\omega_{-1}=\omega_{L}$ yields

$$
\tau_{3}\left(\omega, \omega_{L}, \Omega_{-2}\right)=\left[1+\tau_{1}\left(\omega, \omega_{L}, \Omega_{-2}\right)\right]\left\{\frac{q^{b}\left(\omega_{L}\right) c^{\prime}\left[q\left(\omega_{L}\right)\right] / \alpha_{L}}{q^{b}(\omega) c^{\prime}[q(\omega)] / \alpha}-1\right\},
$$




\section{4}

which gives us the realized money growth rate

$$
\gamma\left(\omega, \omega_{L}, \Omega_{-2}\right)=\left[1+\tau_{1}\left(\omega, \omega_{L}, \Omega_{-2}\right)\right] \frac{q^{b}\left(\omega_{L}\right) c^{\prime}\left[q\left(\omega_{L}\right)\right] / \alpha_{L}}{q^{b}(\omega) c^{\prime}[q(\omega)] / \alpha}
$$

and from the money demand equation we get

$$
1=\beta \int_{\Phi} \frac{\varepsilon u^{\prime}\left[q^{b}(\omega)\right] q^{b}(\omega)}{q^{b}\left(\omega_{L}\right) c^{\prime}\left[q\left(\omega_{L}\right)\right] / \alpha_{L}} \frac{f\left(\omega \mid \omega_{L}, \Omega_{-2}\right)}{1+\tau_{1}\left(\omega, \omega_{L}\right)} d \omega
$$

This equation imposes a restriction on the choice of the vector $\left\{\tau_{1}\left(\omega, \omega_{L}\right)\right\}_{\omega \in \Phi}$. One such vector choice is $\tau_{1}\left(\omega, \omega_{L}\right)=\tau_{1}$ for all $\omega$. This pins down $\tau_{1}\left(\omega_{L}, \omega_{L}\right)$ and requires

$$
\tau_{1}=\beta \int_{\Phi} \frac{\varepsilon u^{\prime}\left[q^{b}(\omega)\right] q^{b}(\omega)}{q^{b}\left(\omega_{L}\right) c^{\prime}\left[q\left(\omega_{L}\right)\right] / \alpha_{L}} f\left(\omega \mid \omega_{L}, \Omega_{-2}\right) d \omega-1
$$

Now consider an arbitrary $\omega_{-1}$. Again we obtain

$$
\tau_{3}\left(\omega, \omega_{-1}, \Omega_{-2}\right)=\left[1+\tau_{1}\left(\omega, \omega_{-1}, \Omega_{-2}\right)\right]\left\{\frac{q^{b}\left(\omega_{L}\right) c^{\prime}\left[q\left(\omega_{L}\right)\right] / \alpha_{L}}{q^{b}(\omega) c^{\prime}[q(\omega)] / \alpha}-1\right\},
$$

which gives us

$$
\gamma\left(\omega, \omega_{-1}, \Omega_{-2}\right)=\left[1+\tau_{1}\left(\omega, \omega_{-1}, \Omega_{-2}\right)\right] \frac{q^{b}\left(\omega_{L}\right) c^{\prime}\left[q\left(\omega_{L}\right)\right] / \alpha_{L}}{q^{b}(\omega) c^{\prime}[q(\omega)] / \alpha},
$$

and the money demand equation is

$$
1=\beta \int_{\Phi}\left\{\frac{\varepsilon u^{\prime}\left[q^{b}(\omega)\right] q^{b}(\omega)}{q^{b}\left(\omega_{L}\right) c^{\prime}\left[q\left(\omega_{L}\right)\right] / \alpha_{L}}\right\} \frac{f\left(\omega \mid \omega_{-1}, \Omega_{-2}\right)}{1+\tau_{1}\left(\omega, \omega_{-1}\right)} d \omega
$$

Thus, for both (A.17) and (A.18) to hold as they do for $\omega_{L}$, we must have

$$
1+\tau_{1}\left(\omega, \omega_{-1}, \Omega_{-2}\right)=\frac{f\left(\omega \mid \omega_{-1}, \Omega_{-2}\right)}{f\left(\omega \mid \omega_{L}, \Omega_{-2}\right)}\left[1+\tau_{1}\left(\omega, \omega_{L}, \Omega_{-2}\right)\right] \quad \forall \omega, \omega_{-1} .
$$

This pins down every transfer as a function of $\tau_{1}\left(\omega, \omega_{L}, \Omega_{-2}\right)$. Thus, for $\tau_{1}\left(\omega, \omega_{L}, \Omega_{-2}\right)=$ $\tau_{1}$, we have the transfer scheme

$$
\begin{aligned}
1+\tau_{1}(\Omega) & =\frac{f\left(\omega \mid \omega_{-1}, \Omega_{-2}\right)}{f\left(\omega \mid \omega_{L}, \Omega_{-2}\right)}\left(1+\tau_{1}\right) \\
\tau_{3}(\Omega) & =\left(1+\tau_{1}\right) \frac{f\left(\omega \mid \omega_{-1}, \Omega_{-2}\right)}{f\left(\omega \mid \omega_{L}, \Omega_{-2}\right)}\left\{\frac{q^{b}\left(\omega_{L}\right) c^{\prime}\left[q\left(\omega_{L}\right)\right] / \alpha_{L}}{q^{b}(\omega) c^{\prime}[q(\omega)] / \alpha}-1\right\}
\end{aligned}
$$

and the stochastic inflation rate

$$
\gamma(\Omega)=\left(1+\tau_{1}\right) \frac{f\left(\omega \mid \omega_{-1}, \Omega_{-2}\right)}{f\left(\omega \mid \omega_{L}, \Omega_{-2}\right)} \frac{q^{b}\left(\omega_{L}\right) c^{\prime}\left[q\left(\omega_{L}\right)\right] / \alpha_{L}}{q^{b}(\omega) c^{\prime}[q(\omega)] / \alpha} .
$$


The remaining endogenous variables are then

$$
\begin{aligned}
\phi(\Omega) & =\frac{q^{b}\left(\omega_{L}\right) c^{\prime}[q(\omega)] / \alpha}{M_{-1}\left(1+\tau_{1}\right)} \frac{f\left(\omega \mid \omega_{-1}, \Omega_{-2}\right)}{f\left(\omega \mid \omega_{L}, \Omega_{-2}\right)} \text { and } p(\Omega) \\
& =M_{-1} \frac{\left(1+\tau_{1}\right) f\left(\omega \mid \omega_{-1}, \Omega_{-2}\right)}{q^{b}(\omega) f\left(\omega \mid \omega_{L}, \Omega_{-2}\right)}
\end{aligned}
$$

Proof of Proposition 2. The proof follows the lines of the proof of Proposition 1.

First step. The central bank allocation has to satisfy two constraints. The first constraint is the entry condition (18), which holds in each state. The second constraint is the pricing equation (27), which also holds in each state. We can use (18) to eliminate $p \phi$ from (27) to get

$$
k\left(s_{t}\right)=\left[c^{\prime}\left(q_{t}\right) q_{t}\left(1-R^{u}\right)^{-1}-c\left(q_{t}\right)\right] / \alpha_{t} .
$$

Notice the appearance of the markup $\left(1-R^{u}\right)^{-1}$, which is absent from (A.5).

The optimal allocation solves

$$
\begin{aligned}
& 0=\frac{\varepsilon_{t} u^{\prime}\left(q_{t}^{b}\right)}{\left(1 / \alpha_{t}\right) c^{\prime}\left[q_{t}\left(\omega_{t}\right)\right]}-1-\lambda_{t} \frac{R^{c}+R^{u}}{1-R^{u}}, \\
& 0=\varepsilon_{t} u^{\prime}\left(q_{t}^{b}\right) q_{t}-c\left(q_{t}\right) / \alpha_{t}-k\left(s_{t}\right)-\left(1-\lambda_{t}\right)\left(1+\Sigma^{\sigma}\right)^{-1} s_{t} k^{\prime}\left(s_{t}\right), \\
& 0=k\left(s_{t}\right)-\left[c^{\prime}\left(q_{t}\right) q_{t}\left(1-R^{u}\right)^{-1}-c(q)\right] / \alpha_{t}, \\
& 0=q_{t}^{b}-s_{t} \sigma\left(s_{t}\right) q_{t} .
\end{aligned}
$$

Note that for $R^{u}=0$, (A.20)-(A.22) and (A.6)-(A.8) are identical. Again, because there are no terms involving past or future values, the solution to (A.20)-(A.22) is independent of $t$ and $\Omega$, so it is stationary. Use (A.20) and (A.22) to write (A.21) as follows:

$$
\lambda=\frac{R^{u}\left(1+\Sigma^{\sigma}\right) c^{\prime}\left(q_{t}\right) q_{t} / \alpha_{t}+\left(1-R^{u}\right) s_{t} k^{\prime}\left(s_{t}\right)}{\left(R^{c}+R^{u}\right)\left(1+\Sigma^{\sigma}\right) c^{\prime}\left(q_{t}\right) q_{t} / \alpha_{t}+\left(1-R^{u}\right) s_{t} k^{\prime}\left(s_{t}\right)} .
$$

Use (A.24) to replace $\lambda$ in (A.20). Then $q$ and $s$ solve

$$
\begin{aligned}
& \frac{\varepsilon u^{\prime}\{[s \sigma(s) / n] q\}}{c^{\prime}(q) / \alpha}-1 \\
&=\left(\frac{R^{c}+R^{u}}{1-R^{u}}\right)\left[\frac{R^{u}\left(1+\Sigma^{\sigma}\right) c^{\prime}\left(q_{t}\right) q_{t} / \alpha_{t}+\left(1-R^{u}\right) s_{t} k^{\prime}\left(s_{t}\right)}{\left(R^{c}+R^{u}\right)\left(1+\Sigma^{\sigma}\right) c^{\prime}\left(q_{t}\right) q_{t} / \alpha_{t}+\left(1-R^{u}\right) s_{t} k^{\prime}\left(s_{t}\right)}\right], \\
& k(s)-\left[c^{\prime}(q) q\left(1-R^{u}\right)^{-1}-c(q)\right] / \alpha=0 .
\end{aligned}
$$

By comparing these two expressions to the first-best allocation (A.3)-(A.4), it is straightforward to show that $q<q^{*}$. To establish that $s \geq s^{*}$, we can replicate the same proof as in the previous case of competitive pricing.

Second step. Because (A.7) and (A.21) are identical and $\lambda<1$, we can replicate the proof of Step 2 of Proposition 2 one for one. 
Third step. We now show that a set of transfers $\tau_{1}(\Omega)$ and $\tau_{3}(\Omega)$ exists that implements the central bank's allocation and satisfies (23). Using (24) and (27), we can write (23) as follows:

$$
1=\beta\left(1-R^{u}\right) \int_{\Phi} \frac{\varepsilon u^{\prime}\{\sigma[s(\omega)] s(\omega) q(\omega)\}}{\gamma(\Omega)(1 / \alpha) c^{\prime}[q(\omega)]} d F\left(\omega \mid \Omega^{-1}\right)
$$

We first consider the case $k^{\prime}(s)=0$. Because $\left(1-R^{u}\right) \varepsilon u^{\prime}\{\sigma[s(\omega)] s(\omega) q(\omega)\}=$ $(1 / \alpha) c^{\prime}[q(\omega)]$ under the central bank's allocation, (A.27) reduces to

$$
1=\beta \int_{\Phi} \frac{1}{\gamma(\Omega)} d F\left(\omega_{t} \mid \Omega_{t-1}\right)=\beta \int_{\Phi} \frac{1}{1+\tau_{1}(\Omega)+\tau_{3}(\Omega)} d F\left(\omega_{t} \mid \Omega_{t-1}\right) .
$$

It is clear that any set of transfers $\tau_{1}(\Omega)$ and $\tau_{3}(\Omega)$ that satisfies $\gamma(\Omega)=1+\tau_{1}(\Omega)+\tau_{3}(\Omega)=$ $\beta$ for all $\omega=\Phi$ implements the central bank's allocation.

Consider next the case $k^{\prime}(s)>0$. Assume that the transfers are such that the agents have just enough money to buy $q^{b}(\omega)$ in each state; i.e., $p(\Omega) q^{b}(\omega)=M_{-1}\left[1+\tau_{1}(\Omega)\right]$. From the pricing equation (27), we can write this expression as follows:

$$
\left(1-R^{u}\right)^{-1} q^{b}(\omega) c^{\prime}[q(\omega)] / \alpha=\phi(\Omega) M_{-1}\left[1+\tau_{1}(\Omega)\right] .
$$

Because $z=\phi(\Omega) M_{-1}\left[1+\tau_{1}(\Omega)+\tau_{3}(\Omega)\right]$, using (A.15), we get (A.16). As before, assume the central bank mainly conditions the money growth rate on the shocks at $t$ and $t-1$. Consider the state $\omega_{L}=\left(\alpha_{L}, \varepsilon_{L}\right)$ and set $\tau_{3}\left(\omega_{L}, \omega_{L}\right)=0$. Thus, $z=(1-$ $\left.R^{u}\right)^{-1} q^{b}\left(\omega_{L}\right) c^{\prime}\left[q\left(\omega_{L}\right)\right] / \alpha_{L}$ is the real stock of money. We then have

$$
\left(1-R^{u}\right)^{-1} q^{b}\left(\omega_{L}\right) c^{\prime}\left[q\left(\omega_{L}\right)\right] / \alpha_{L}=\phi(\Omega) M_{-1}\left[1+\tau_{1}(\Omega)+\tau_{3}(\Omega)\right] .
$$

Using this expression and (A.28), we can obtain $\tau_{3}\left(\omega, \omega_{L}\right)$ as a function of $\tau_{1}\left(\omega, \omega_{L}\right)$ :

$$
\tau_{3}\left(\omega, \omega_{L}, \Omega_{-2}\right)=\left[1+\tau_{1}\left(\omega, \omega_{L}, \Omega_{-2}\right)\right]\left\{\frac{q^{b}\left(\omega_{L}\right) c^{\prime}\left[q\left(\omega_{L}\right)\right] / \alpha_{L}}{q^{b}(\omega) c^{\prime}[q(\omega)] / \alpha}-1\right\} .
$$

The realized money growth satisfies

$$
\gamma\left(\omega, \omega_{L}, \Omega_{-2}\right)=\left[1+\tau_{1}\left(\omega, \omega_{L}, \Omega_{-2}\right)\right] \frac{q^{b}\left(\omega_{L}\right) c^{\prime}\left[q\left(\omega_{L}\right)\right] / \alpha_{L}}{q^{b}(\omega) c^{\prime}[q(\omega)] / \alpha} .
$$

Then replace $\gamma\left(\omega, \omega_{L}, \Omega_{-2}\right)$ in (A.14) to get

$$
1=\beta\left(1-R^{u}\right) \int_{\Phi} \frac{q^{b}(\omega) \varepsilon u^{\prime}\left[q^{b}(\omega)\right]}{q^{b}\left(\omega_{L}\right) c^{\prime}\left[q\left(\omega_{L}\right)\right] / \alpha_{L}} \frac{f\left(\omega \mid \omega_{L}, \Omega_{-2}\right)}{1+\tau_{1}\left(\omega, \omega_{L}, \Omega_{-2}\right)} d \omega .
$$

This equation imposes a restriction on the vector $\left\{\tau_{1}\left(\omega, \omega_{L}, \Omega_{-2}\right)\right\}_{\omega \in \Phi}$. However, there are many choices that are consistent with this equation. One particular choice is $\tau_{1}\left(\omega, \omega_{L}, \Omega_{-2}\right)=\tau_{1}$, in which case we have the transfer scheme

$$
\begin{aligned}
\tau_{1} & =\beta\left(1-R^{u}\right) \int_{\Phi} \frac{q^{b}(\omega) \varepsilon u^{\prime}\left[q^{b}(\omega)\right]}{q^{b}\left(\omega_{L}\right) c^{\prime}\left[q\left(\omega_{L}\right)\right] / \alpha_{L}} f\left(\omega \mid \omega_{L}, \Omega_{-2}\right) d \omega-1, \\
\tau_{3}\left(\omega, \omega_{L}, \Omega_{-2}\right) & =\left(1+\tau_{1}\right)\left\{\frac{q^{b}\left(\omega_{L}\right) c^{\prime}\left[q\left(\omega_{L}\right)\right] / \alpha_{L}}{q^{b}(\omega) c^{\prime}[q(\omega)] / \alpha}-1\right\} .
\end{aligned}
$$


Now pick an arbitrary state $\omega_{-1}$. Once again we obtain

$$
\begin{aligned}
& \tau_{3}\left(\omega, \omega_{-1}, \Omega_{-2}\right)=\left[1+\tau_{1}\left(\omega, \omega_{-1}, \Omega_{-2}\right)\right]\left\{\frac{q^{b}\left(\omega_{L}\right) c^{\prime}\left[q\left(\omega_{L}\right)\right] / \alpha_{L}}{q^{b}(\omega) c^{\prime}[q(\omega)] / \alpha}-1\right\}, \\
& \gamma\left(\omega, \omega_{-1}, \Omega_{-2}\right)=\left[1+\tau_{1}\left(\omega, \omega_{-1}, \Omega_{-2}\right)\right] \frac{q^{b}\left(\omega_{L}\right) c^{\prime}\left[q\left(\omega_{L}\right)\right] / \alpha_{L}}{q^{b}(\omega) c^{\prime}[q(\omega)] / \alpha}
\end{aligned}
$$

and the money demand equation

$$
1=\beta\left(1-R^{u}\right) \int_{\Phi} \frac{q^{b}(\omega) \varepsilon u^{\prime}\left[q^{b}(\omega)\right]}{q^{b}\left(\omega_{L}\right) c^{\prime}\left[q\left(\omega_{L}\right)\right] / \alpha_{L}} \frac{f\left(\omega \mid \omega_{-1}, \Omega_{-2}\right)}{1+\tau_{1}\left(\omega, \omega_{-1}, \Omega_{-2}\right)} d \omega
$$

Again, for the money demand equations (A.31) and (A.32) to hold, we must have

$$
1+\tau_{1}\left(\omega, \omega_{-1}, \Omega_{-2}\right)=\left(1+\tau_{1}\right) \frac{f\left(\omega \mid \omega_{-1}, \Omega_{-2}\right)}{f\left(\omega \mid \omega_{L}, \Omega_{-2}\right)}
$$

which implies that the inflation rate is given by

$$
\gamma(\Omega)=\left(1+\tau_{1}\right) \frac{f\left(\omega \mid \omega_{-1}, \Omega_{-2}\right)}{f\left(\omega \mid \omega_{L}, \Omega_{-2}\right)} \frac{q^{b}\left(\omega_{L}\right) c^{\prime}\left[q\left(\omega_{L}\right)\right] / \alpha_{L}}{q^{b}(\omega) c^{\prime}[q(\omega)] / \alpha},
$$

and the price of money and the price of goods are stochastic and satisfy

$$
\begin{aligned}
\phi(\Omega) & =\frac{f\left(\omega \mid \omega_{L}, \Omega_{-2}\right) q^{b}(\omega) c^{\prime}[q(\omega)] / \alpha}{f\left(\omega \mid \omega_{-1}, \Omega_{-2}\right)\left(1-R^{u}\right) M_{-1}\left(1+\tau_{1}\right)}, \\
p(\Omega) & =M_{-1} \frac{\left(1+\tau_{1}\right) f\left(\omega \mid \omega_{-1}, \Omega_{-2}\right)}{q^{b}(\omega) f\left(\omega \mid \omega_{L}, \Omega_{-2}\right)} .
\end{aligned}
$$

Proof of Proposition 3. In this proof, we show that it is optimal and feasible to implement the same allocation as for state-contingent monopoly pricing. The central bank allocation has to satisfy the entry condition (18) and the pricing equation (29). Let $\hat{\phi}\left(\Omega_{t}\right) \equiv \phi\left(\Omega_{t}\right) p\left(\Omega_{t-1}\right)$, which is the relative price between market 2 and market 3 goods. Because at time $t, p\left(\Omega_{t-1}\right)$ is a predetermined variable, the central bank can affect this relative price by changing $\phi\left(\Omega_{t}\right)$ via policy. Then rewrite (29) as

$$
\int_{\Phi} \sigma\left(s_{t}\right) s_{t} q_{t}\left[\hat{\phi}_{t}\left(1-R^{u}\right)-c^{\prime}\left(q_{t}\right) / \alpha_{t}\right] d F\left(\omega_{t} \mid \Omega_{t-1}\right)=0
$$

The central bank now chooses $q^{b}, q, s$, and $\hat{\phi}$ to maximize 


$$
\begin{aligned}
L_{p} & =\sum_{t=0}^{\infty} \beta^{t} \int_{\Phi}\left[\varepsilon_{t} u\left(q_{t}^{b}\right)-\sigma\left(s_{t}\right) s_{t} c\left(q_{t}\right) / \alpha_{t}\right] d F\left(\omega_{t} \mid \Omega_{t-1}\right) \\
& -\sum_{t=0}^{\infty} \beta^{t} \int_{\Phi} s_{t} k\left(s_{t}\right) d F\left(\omega_{t} \mid \Omega_{t-1}\right) \\
& +\sum_{t=0}^{\infty} \beta^{t} \int_{\Phi} \mu_{t}\left(s_{t} q_{t}-q_{t}^{b}\right) d F\left(\omega_{t} \mid \Omega_{t-1}\right) \\
& +\sum_{t=0}^{\infty} \beta^{t} \int_{\Phi} \hat{\lambda}_{t}\left\{k\left(s_{t}\right)-\left[\hat{\phi}_{t} q_{t}-c\left(q_{t}\right)\right] / \alpha_{t}\right\} d F\left(\omega_{t} \mid \Omega_{t-1}\right) \\
& +\sum_{t=0}^{\infty} \beta^{t} \theta_{t} \int_{\Phi} \sigma\left(s_{t}\right) s_{t} q_{t}\left[\hat{\phi}_{t}\left(1-R^{u}\right)-c^{\prime}\left(q_{t}\right) / \alpha_{t}\right] d F\left(\omega_{t} \mid \Omega_{t-1}\right)
\end{aligned}
$$

where $\hat{\lambda}_{t}=\lambda_{t} \sigma\left(s_{t}\right) s_{t}$ is the Lagrange multiplier for (18) and $\theta_{t}$ is the one for (29). Because we know that $s_{t} q_{t}=n_{t} q_{t}^{b}$ in equilibrium, we can eliminate $q_{t}^{b}$ and simply choose $q_{t}, s_{t}$ and $\hat{\phi}_{t}$ for all $t$. The first-order conditions for $q_{t}, s_{t}$, and $\hat{\phi}_{t}$ are

$$
\begin{gathered}
0=\varepsilon_{t} u^{\prime}\left[\left(\sigma\left(s_{t}\right) s_{t} q_{t}\right)\right]-c^{\prime}\left(q_{t}\right) / \alpha_{t}-\lambda_{t}\left(\frac{R^{u}+R^{c}}{1-R^{u}}\right) c^{\prime}\left(q_{t}\right) / \alpha_{t}, \\
\lambda_{t}\left[\hat{\phi}_{t}-\frac{c^{\prime}\left(q_{t}\right) / \alpha_{t}}{1-R^{u}}\right] \\
=\varepsilon_{t} u^{\prime}\left[\left(\sigma\left(s_{t}\right) s_{t} q_{t}\right)\right] q_{t}-c^{\prime}\left(q_{t}\right) / \alpha_{t}-k\left(s_{t}\right)-s_{t} k^{\prime}\left(s_{t}\right)\left(1-\lambda_{t}\right), \\
0=k\left(s_{t}\right)-\hat{\phi}_{t} q_{t}+c\left(q_{t}\right) / \alpha_{t} .
\end{gathered}
$$

If the central bank enacts a policy such that the relative price is given by

$$
\hat{\phi}(\Omega) \equiv \phi(\Omega) p\left(\Omega_{-1}\right)=\frac{c^{\prime}[q(\omega)] / \alpha}{1-R^{u}}
$$

then (A.33)-(A.35) reduce to (A.20)-(A.22). Furthermore, this choice also satisfies the pricing equation (29). Consequently, the central bank chooses the same allocation as with state-contingent monopoly pricing.

Implementation: The central bank wants to replicate the state-contingent monopoly pricing allocation. All that remains to be determined is how to implement it with non-statecontingent pricing. Consider the case $k^{\prime}(s)>0$. Assume that the transfers are such that the agents have just enough money to buy $q(\omega)$ in each state. This implies that the aggregate money stock must purchase total nominal output in market 2 ; i.e., $M_{-1}\left[1+\tau_{1}(\Omega)\right]=$ $p\left(\Omega_{-1}\right) q^{b}(\omega)$. From (A.36), we have

$$
\phi(\Omega) M_{-1}\left[1+\tau_{1}(\Omega)\right]=\left(1-R^{u}\right)^{-1} q^{b}(\omega) c^{\prime}[q(\omega)] / \alpha .
$$

We also have $z=\phi(\Omega) M_{-1}\left[1+\tau_{1}(\Omega)+\tau_{3}(\Omega)\right]$. Using (A.37), we get

$$
z=\left(1-R^{u}\right)^{-1} q^{b}(\omega) c^{\prime}[q(\omega)] / \alpha+\tau_{3}(\Omega) \phi(\Omega) M_{-1} .
$$


As before, assume that the central bank only conditions the money growth rate on the last two shocks for any $\Omega_{-2}$. Let $\phi(\Omega)=\phi\left(\omega, \omega_{-1}, \Omega_{-2}\right)$. Consider the state $\omega_{L}=\left(\alpha_{L}, \varepsilon_{L}\right)$ and set $\tau_{3}\left(\omega_{L}, \omega_{L}\right)=0$. Then from (A.38) we have

$$
z=\left(1-R^{u}\right)^{-1} q^{b}\left(\omega_{L}\right) c^{\prime}\left[q\left(\omega_{L}\right)\right] / \alpha_{L} .
$$

This pins down the real stock of money. It then follows from the buyer's budget constraint that

$$
1+\tau_{1}\left(\omega, \omega_{L}, \Omega_{-2}\right)=\left[1+\tau_{1}\left(\omega_{L}, \omega_{L}, \Omega_{-2}\right)\right] \frac{q^{b}(\omega)}{q^{b}\left(\omega_{L}\right)} .
$$

In short, with $p\left(\Omega_{-1}\right)$ fixed, nominal spending has to rise as $q^{b}(\omega)$ increases, meaning that the nominal injection in market 1 must also rise, regardless of what happens in market 3. We can then solve for $\tau_{3}\left(\omega, \omega_{L}\right)$ as before to obtain (A.29). Using the preceding expression in (A.29) yields

$$
\tau_{3}\left(\omega, \omega_{L}, \Omega_{-2}\right)=\left[1+\tau_{1}\left(\omega_{L}, \omega_{L}, \Omega_{-2}\right)\right]\left\{\frac{c^{\prime}\left[q\left(\omega_{L}\right)\right] / \alpha_{L}}{c^{\prime}[q(\omega)] / \alpha}-\frac{q^{b}(\omega)}{q^{b}\left(\omega_{L}\right)}\right\},
$$

so

$$
\gamma\left(\omega, \omega_{L}, \Omega_{-2}\right)=\left[1+\tau_{1}\left(\omega_{L}, \omega_{L}, \Omega_{-2}\right)\right] \frac{c^{\prime}\left[q\left(\omega_{L}\right)\right] / \alpha_{L}}{c^{\prime}[q(\omega)] / \alpha}
$$

Using this expression and (A.36) in (23) we obtain

$$
1=\beta\left(1-R^{u}\right) \int_{\Phi} \frac{\varepsilon u^{\prime}\left[q^{b}(\omega)\right]}{c^{\prime}\left[q\left(\omega_{L}\right)\right] / \alpha_{L}} \frac{f\left(\omega \mid \omega_{L}, \Omega_{-2}\right)}{1+\tau_{1}\left(\omega_{L}, \omega_{L}, \Omega_{-2}\right)} d \omega .
$$

This places a restriction on $\tau_{1}\left(\omega_{L}, \omega_{L}, \Omega_{-2}\right)$ given by

$$
\tau_{1}\left(\omega_{L}, \omega_{L}, \Omega_{-2}\right)=\beta\left(1-R^{u}\right) \int_{\Phi} \frac{\varepsilon u^{\prime}\left[q^{b}(\omega)\right]}{c^{\prime}\left[q\left(\omega_{L}\right)\right] / \alpha_{L}} f\left(\omega \mid \omega_{L}, \Omega_{-2}\right) d \omega-1 .
$$

This gives us all of the transfers for $\omega_{-1}=\omega_{L}$.

Now consider any state $\omega_{-1}$. We get

$$
\begin{aligned}
& \tau_{3}\left(\omega, \omega_{-1}, \Omega_{-2}\right)=\left[1+\tau_{1}\left(\omega, \omega_{-1}, \Omega_{-2}\right)\right]\left[\frac{q^{b}\left(\omega_{L}\right) c^{\prime}\left[q\left(\omega_{L}\right)\right] / \alpha_{L}}{q^{b}(\omega) c^{\prime}[q(\omega)] / \alpha}-1\right] \\
& \gamma\left(\omega, \omega_{-1}, \Omega_{-2}\right)=\left[1+\tau_{1}\left(\omega, \omega_{-1}, \Omega_{-2}\right)\right] \frac{q^{b}\left(\omega_{L}\right) c^{\prime}\left[q\left(\omega_{L}\right)\right] / \alpha_{L}}{q^{b}(\omega) c^{\prime}[q(\omega)] / \alpha} .
\end{aligned}
$$

Then from (A.36) in (23) we get

$$
1=\beta\left(1-R^{u}\right) \int_{\Phi} \frac{q^{b}(\omega) \varepsilon u^{\prime}\left[q^{b}(\omega)\right]}{q^{b}\left(\omega_{L}\right) c^{\prime}\left[q\left(\omega_{L}\right)\right] / \alpha_{L}} \frac{f\left(\omega \mid \omega_{-1}, \Omega_{-2}\right)}{1+\tau_{1}\left(\omega, \omega_{-1}, \Omega_{-2}\right)} d \omega .
$$

In order for (A.39) and (A.40) to hold, we must have

$$
\left[1+\tau_{1}\left(\omega, \omega_{-1}, \Omega_{-2}\right)\right]=\left[1+\tau_{1}\left(\omega_{L}, \omega_{L}, \Omega_{-2}\right)\right] \frac{q^{b}(\omega) f\left(\omega \mid \omega_{-1}, \Omega_{-2}\right)}{q^{b}\left(\omega_{L}\right) f\left(\omega \mid \omega_{L}, \Omega_{-2}\right)}
$$


This pins down $\tau_{1}\left(\omega, \omega_{-1}\right)$ as a function of $\tau_{1}\left(\omega_{L}, \omega_{L}\right)$ for all $\omega_{-1}$. Thus the implementation scheme

$\tau_{1}\left(\omega_{L}, \omega_{L}, \Omega_{-2}\right)=\beta\left(1-R^{u}\right) \int_{\Phi} \frac{\varepsilon u^{\prime}\left[q^{b}(\omega)\right]}{c^{\prime}\left[q\left(\omega_{L}\right)\right] / \alpha_{L}} f\left(\omega \mid \omega_{L}, \Omega_{-2}\right) d \omega-1$,

$\tau_{1}\left(\omega, \omega_{-1}, \Omega_{-2}\right)=\left[1+\tau_{1}\left(\omega_{L}, \omega_{L}, \Omega_{-2}\right)\right] \frac{q^{b}(\omega) f\left(\omega \mid \omega_{-1}, \Omega_{-2}\right)}{q^{b}\left(\omega_{L}\right) f\left(\omega \mid \omega_{L}, \Omega_{-2}\right)}-1$,

$\tau_{3}\left(\omega, \omega_{-1}, \Omega_{-2}\right)=\left[1+\tau_{1}\left(\omega_{L}, \omega_{L}, \Omega_{-2}\right)\right] \frac{f\left(\omega \mid \omega_{-1}, \Omega_{-2}\right)}{f\left(\omega \mid \omega_{L}, \Omega_{-2}\right)}\left[\frac{c^{\prime}\left[q\left(\omega_{L}\right)\right] / \alpha_{L}}{c^{\prime}[q(\omega)] / \alpha}-\frac{q^{b}(\omega)}{q^{b}\left(\omega_{L}\right)}\right]$,

and the subsequent inflation rates,

$$
\begin{aligned}
\gamma\left(\omega, \omega_{L}, \Omega_{-2}\right) & =\left[1+\tau_{1}\left(\omega_{L}, \omega_{L}, \Omega_{-2}\right)\right] \frac{c^{\prime}\left[q\left(\omega_{L}\right)\right] / \alpha_{L}}{c^{\prime}[q(\omega)] / \alpha} \quad \forall \omega \\
\gamma(\Omega) & =\gamma\left(\omega, \omega_{L}, \Omega_{-2}\right) \frac{f\left(\omega \mid \omega_{-1}, \Omega_{-2}\right)}{f\left(\omega \mid \omega_{L}, \Omega_{-2}\right)}
\end{aligned}
$$

allow the central bank to implement the state-contingent monopoly pricing allocation even though there is price posting. We can then solve for the equilibrium prices

$$
\begin{aligned}
\phi(\Omega) & =\frac{q^{b}(\omega) c^{\prime}[q(\omega)] / \alpha}{M_{-1}\left[1+\tau_{1}\left(\omega, \omega_{-1}, \Omega_{-2}\right)\right]\left(1-R^{u}\right)} \text { and } \\
p\left(\Omega_{-1}\right) & =M_{-1} \frac{\left[1+\tau_{1}\left(\omega_{L}, \omega_{L}, \Omega_{-2}\right)\right] f\left(\omega \mid \omega_{-1}, \Omega_{-2}\right)}{q^{b}\left(\omega_{L}\right) f\left(\omega \mid \omega_{L}, \Omega_{-2}\right)} .
\end{aligned}
$$

